\title{
Theoretical Study of Nucleophilic Substitution at Sulfur in Sulfinyl Derivatives
}

\author{
Steven H. Norton, Steven M. Bachrach*, and Joseph M. Hayes \\ Department of Chemistry \\ Trinity University \\ One Trinity Place \\ San Antonio, TX 78212 \\ sbachrach@trinity.edu
}

\begin{abstract}
Supporting Information Available: Relative energies of the critical points for Reactions 1-6 at HF/6-31+G*, MP2/6-31G*, B3LYP/6-31G*, and B3LYP/aug-cc-pVDZ (Table S1) along with the coordinates of all optimized structures, their absolute energies, and number of imaginary frequencies. This material is available free of charge via the Internet at http://pubs.acs.org.

\section{Page $\quad$ Contents}

S2-S3.........Table S1. Relative energies of critical points along the PES for reactions 1-6. S4-S18.....HF/6-31+G*, MP2/6-31G*, B3LYP/6-31G*, and B3LYP/aug-cc-pVDZ coordinates of all optimized structures
\end{abstract}


Table S1. Relative energies ( $\mathrm{kcal} \mathrm{mol}^{-1}$ ) of critical points along the PES for reactions 1-6. (Top entry is $\Delta E$ and bottom entry is $\Delta G$.)

\begin{tabular}{|c|c|c|c|c|c|}
\hline $\operatorname{rxn} 1 \mathrm{a}$ & $\mathbf{R}$ & IDC & TS & INT & \\
\hline \multirow[t]{2}{*}{$\mathrm{HF} / 6-31+\mathrm{G}^{*}$} & 0.0 & -16.03 & $-15.44^{a}$ & $b$ & \\
\hline & 0.0 & -9.66 & $-8.10^{a}$ & $b$ & \\
\hline \multirow[t]{2}{*}{$\operatorname{MP2}(\mathrm{FC}) / 6-31+\mathrm{G}^{*}$} & 0.0 & $c$ & $c$ & -24.76 & \\
\hline & 0.0 & $c$ & $c$ & -17.68 & \\
\hline \multirow[t]{2}{*}{ B3L/6-31+G* } & 0.0 & -16.80 & -16.81 & -26.17 & \\
\hline & 0.0 & -11.63 & -10.39 & -19.08 & \\
\hline \multirow[t]{2}{*}{ B3L/aug-cc-pVDZ } & 0.0 & -17.36 & -17.31 & -26.43 & \\
\hline & 0.0 & -12.05 & -10.86 & -19.29 & \\
\hline $\operatorname{rxn} 1 \mathrm{~b}$ & $\mathbf{R}$ & IDC & TS & INT & \\
\hline \multirow[t]{2}{*}{$\mathrm{HF} / 6-31+\mathrm{G}^{*}$} & 0.0 & -20.71 & -20.15 & -20.75 & \\
\hline & 0.0 & -14.65 & -13.24 & -15.15 & \\
\hline \multirow[t]{2}{*}{$\operatorname{MP2}(\mathrm{FC}) / 6-31+\mathrm{G}^{*}$} & 0.0 & -25.84 & -24.07 & -28.54 & \\
\hline & 0.0 & -20.22 & -17.64 & -21.61 & \\
\hline \multirow[t]{2}{*}{ B3L/6-31+G* } & 0.0 & -27.70 & -25.11 & -31.33 & \\
\hline & 0.0 & -23.09 & -19.04 & -24.32 & \\
\hline \multirow[t]{2}{*}{ B3L/aug-cc-pVDZ } & 0.0 & -29.60 & -25.83 & -31.53 & \\
\hline & 0.0 & -24.99 & -19.66 & -24.42 & \\
\hline $\operatorname{rxn} 1 \mathrm{c}$ & $\mathbf{R}$ & IDC & TS & INT & \\
\hline \multirow[t]{2}{*}{$\mathrm{HF} / 6-31+\mathrm{G}^{*}$} & 0.0 & -11.34 & -11.33 & - & \\
\hline & 0.0 & -5.35 & -4.23 & - & \\
\hline \multirow[t]{2}{*}{ MP2(FC)/6-31+G* } & 0.0 & -17.81 & -17.71 & -24.48 & \\
\hline & 0.0 & -10.80 & -9.17 & -17.53 & \\
\hline \multirow[t]{2}{*}{ B3L/6-31+G* } & 0.0 & -14.83 & -14.36 & -23.33 & \\
\hline & 0.0 & -8.61 & -7.29 & -16.40 & \\
\hline \multirow[t]{2}{*}{ B3L/aug-cc-pVDZ } & 0.0 & -14.87 & -14.38 & -23.81 & \\
\hline & 0.0 & -8.68 & -7.45 & -16.72 & \\
\hline $\operatorname{rxn} 2$ & $\mathbf{R}$ & IDC & TS & INT & $\mathbf{T S 2}^{a}$ \\
\hline \multirow[t]{2}{*}{$\mathrm{HF} / 6-31+\mathrm{G}^{*}$} & 0.0 & -15.85 & $b$ & $b$ & -6.35 \\
\hline & 0.0 & -8.90 & $b$ & $b$ & 3.25 \\
\hline \multirow[t]{2}{*}{$\operatorname{MP2}(\mathrm{FC}) / 6-31+\mathrm{G}^{*}$} & 0.0 & -18.29 & $b$ & $b$ & -16.30 \\
\hline & 0.0 & -13.70 & $b$ & $b$ & -10.45 \\
\hline \multirow[t]{2}{*}{ B3L/6-31+G* } & 0.0 & -16.52 & -16.50 & $-19.12^{d}$ & -19.07 \\
\hline & 0.0 & -9.34 & -8.22 & $-10.89 d$ & -10.22 \\
\hline \multirow[t]{2}{*}{ B3L/aug-cc-pVDZ } & 0.0 & -17.16 & -17.15 & $-20.21^{d}$ & -20.21 \\
\hline & 0.0 & -10.33 & -9.06 & $-12.84^{d}$ & -11.41 \\
\hline $\operatorname{rxn} 3$ & $\mathbf{R}$ & IDC & $\mathbf{T S}$ & INT & \\
\hline $\mathrm{HF} / 6-31+\mathrm{G}^{*}$ & 0.0 & -13.19 & $6.87^{a}$ & $b$ & \\
\hline
\end{tabular}




\begin{tabular}{|c|c|c|c|c|c|}
\hline & 0.0 & -5.40 & $16.50^{a}$ & $b$ & \\
\hline \multirow[t]{2}{*}{$\mathrm{MP2}(\mathrm{FC}) / 6-31+\mathrm{G}^{*}$} & 0.0 & -17.81 & -9.22 & -13.96 & \\
\hline & 0.0 & -7.62 & -1.46 & -5.40 & \\
\hline \multirow[t]{2}{*}{ B3L/6-31+G* } & 0.0 & -15.78 & -10.83 & -15.18 & \\
\hline & 0.0 & -5.91 & -2.33 & -6.34 & \\
\hline \multirow[t]{2}{*}{ B3L/aug-cc-pVDZ } & 0.0 & -15.56 & -10.77 & -14.19 & \\
\hline & 0.0 & -6.24 & -2.58 & -5.85 & \\
\hline $\operatorname{rxn} 4$ & $\mathbf{R}$ & IDC & TS & INT & \\
\hline \multirow[t]{2}{*}{$\operatorname{MP2}(\mathrm{FC}) / 6-31+\mathrm{G}^{*}$} & 0.0 & -18.97 & -18.97 & -33.23 & \\
\hline & 0.0 & -10.47 & -9.50 & -20.55 & \\
\hline \multirow[t]{2}{*}{ B3L/6-31+G* } & 0.0 & -18.17 & -17.75 & -28.95 & \\
\hline & 0.0 & -9.56 & -8.24 & -16.72 & \\
\hline \multirow[t]{2}{*}{ B3L/aug-cc-pVDZ } & 0.0 & $e$ & -17.78 & -29.16 & \\
\hline & 0.0 & $e$ & -8.20 & -16.50 & \\
\hline $\operatorname{rxn} 5$ & $\mathbf{R}$ & IDC & TS & INT & TS2 $f$ \\
\hline \multirow[t]{2}{*}{$\operatorname{MP2}(\mathrm{FC}) / 6-31+\mathrm{G}^{*}$} & 0.0 & -22.13 & -22.11 & $-34.35^{d}$ & -34.10 \\
\hline & 0.0 & -14.92 & -13.83 & $-24.60^{d}$ & -23.73 \\
\hline \multirow[t]{2}{*}{ B3L/6-31+G* } & 0.0 & $e$ & -21.79 & $-34.49^{d}$ & -34.44 \\
\hline & 0.0 & $e$ & -13.92 & $-24.87 d$ & -24.15 \\
\hline \multirow[t]{2}{*}{ B3L/aug-cc-pVDZ } & 0.0 & $e$ & -21.53 & $-33.32 g$ & $g$ \\
\hline & 0.0 & $e$ & -13.86 & $-23.68 g$ & $g$ \\
\hline $\operatorname{rxn} 6$ & $\mathbf{R}$ & IDC & TS & INT & \\
\hline \multirow[t]{2}{*}{$\operatorname{MP2}(\mathrm{FC}) / 6-31+\mathrm{G}^{*}$} & 0.0 & -18.05 & -17.54 & -25.84 & \\
\hline & 0.0 & -9.78 & -7.53 & -13.62 & \\
\hline \multirow[t]{2}{*}{ B3L/6-31+G* } & 0.0 & -17.67 & -16.66 & -25.34 & \\
\hline & 0.0 & -9.99 & -7.06 & -13.57 & \\
\hline \multirow[t]{2}{*}{ B3L/aug-cc-pVDZ } & 0.0 & $e$ & -15.96 & -23.69 & \\
\hline & 0.0 & $e$ & -6.89 & -12.60 & \\
\hline
\end{tabular}

R: reactants; IDC: ion-dipole complex; TS: transition state; INT: intermediate (tetracoordinate) complex; ${ }^{\mathrm{a}} \mathrm{C}_{\mathrm{S}}$-symmetric $\mathrm{TS}$ for $\mathrm{S}_{\mathrm{N}} 2$ displacement; ${ }^{\mathrm{b}}$ A double-well PES with no tetracoordinate complex was found.; c Only a minimum was found-there appears to be no other critical point.; $\mathrm{d}$ The intermediate complex in this case is not symmetrical $\left(\mathrm{C}_{1}\right)$; see Fig. 2 and discussion in text.; e Proton transfer occurs, IDC not found.. ${ }^{\mathrm{f}} \mathrm{C}_{\mathrm{s}}$-symmetric TS for methyl group rotation; $\mathrm{g}$ The INT is $\mathrm{C}_{\mathrm{s}}$-symmetric in this case, as in almost all others, so there is no TS for methyl group rotation along the reaction coordinate of interest. 


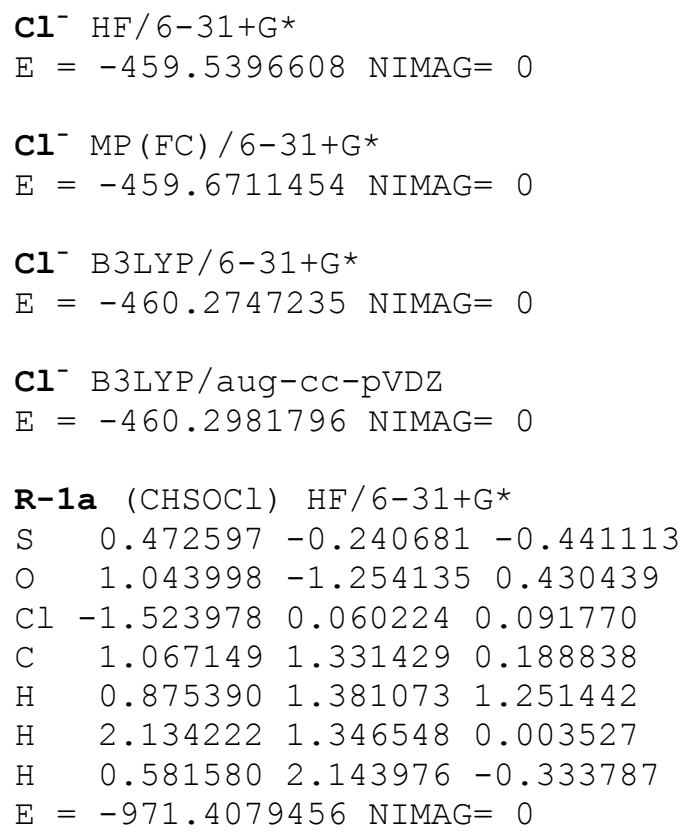

R-1a (CHSOCl) B3LYP/aug-cC-pVDZ

S $\quad 0.516737-0.235139-0.459353$

O $\quad 1.159501-1.248028 \quad 0.452163$

Cl $-1.597762 \quad 0.017127 \quad 0.093382$

C $\quad 1.033069 \quad 1.399041 \quad 0.199893$

$\mathrm{H} \quad 0.820862 \quad 1.4163661 .273715$

$\mathrm{H} \quad 2.112197 \quad 1.462775 \quad 0.009349$

$\mathrm{H} \quad 0.486678 \quad 2.181892-0.337574$

$\mathrm{E}=-973.5478846 \mathrm{NIMAG}=0$
IDC-1a $\mathrm{HF} / 6-31+\mathrm{G}$ *

S $\quad 0.277451 \quad 0.330025-0.159310$

O $\quad 0.455203 \quad 1.702765 \quad 0.264909$

$\mathrm{Cl}-2.806034-0.263752-0.335803$

Cl $2.342016-0.509175-0.407375$

C $\quad-0.000023-0.585724 \quad 1.360117$

H $\quad 0.799108-0.343947 \quad 2.045684$

$\mathrm{H} \quad-0.963320 \quad-0.263074 \quad 1.722554$

$\mathrm{H} \quad-0.028187-1.641401 \quad 1.134769$

$\mathrm{E}=-1430.9731469 \mathrm{NIMAG}=0$

IDC-1a B3LYP/6-31+G*

S $\quad 0.704721 \quad 0.466509 \quad-0.353486$

O $\quad 0.872908 \quad 1.778046 \quad 0.340139$

Cl $-3.524047-0.219389-0.136507$

Cl $2.654886-0.721840-0.097358$

C $\quad-0.315677-0.566678 \quad 0.705695$

$\mathrm{H} \quad-0.050223-0.374202 \quad 1.748442$

$\mathrm{H} \quad-1.382954 \quad-0.300376 \quad 0.492551$

$\mathrm{H} \quad-0.155816-1.6129790 .435201$

$\mathrm{E}=-1433.7920101 \mathrm{NIMAG}=0$

IDC-1a B3LYP/aug-cC-pVDZ

S $\quad 0.694213 \quad 0.459234-0.361733$

$\begin{array}{llll}0 & 0.858309 & 1.786692 & 0.341543\end{array}$

Cl $-3.488116-0.221770-0.131703$

Cl $2.649282-0.719151-0.085924$

C $\quad-0.341268-0.564487 \quad 0.691911$

$\mathrm{H} \quad-0.092154-0.351279 \quad 1.737261$

$\mathrm{H} \quad-1.409441-0.295032 \quad 0.439996$

$\mathrm{H} \quad-0.164514-1.612402 \quad 0.426323$

$\mathrm{E}=-1433.8737312 \mathrm{NIMAG}=0$

TS-1a B3LYP/6-31+G*

S $\quad 0.654523 \quad 0.447381-0.328246$

$\begin{array}{lllll}0 & 0.832386 & 1.776614 & 0.326976\end{array}$

$\mathrm{Cl}-3.459419-0.221082-0.168102$

Cl $2.636925-0.694710-0.142102$

C $\quad-0.284339-0.580750 \quad 0.813132$

H $\quad 0.027567-0.346221 \quad 1.834162$

$\mathrm{H} \quad-1.360768-0.341231 \quad 0.629737$

$\mathrm{H} \quad-0.109827-1.630590 \quad 0.566920$

$\mathrm{E}=-1433.7920192 \mathrm{NIMAG}=1$

TS-1a B3LYP/aug-cC-pVDZ

S $\quad 0.594671 \quad 0.421016-0.310355$

$\begin{array}{lllll}0 & 0.772027 & 1.781342 & 0.320474\end{array}$

Cl $-3.357953-0.224287-0.194557$

Cl $\quad 2.612150-0.662153-0.174811$

C $\quad-0.275192 \quad-0.597090 \quad 0.899794$

H $\quad 0.068234-0.308469 \quad 1.899280$

$\mathrm{H} \quad-1.360786-0.378258 \quad 0.711133$

$\mathrm{H} \quad-0.068606-1.648248 \quad 0.671958$

$E=-1433.8736432 \mathrm{NIMAG}=1$ 
TS-1a $\mathrm{HF} / 6-31+\mathrm{G}$ *

S $\quad 0.000000 \quad 0.3076990 .000000$

O $\quad 1.0601831 .2808820 .000000$

$\mathrm{Cl}-0.488193-0.081762 \quad 2.499224$

Cl $-0.488193-0.081762-2.499224$

C $\quad 0.850187-1.280923 \quad 0.000000$

$\mathrm{H} \quad 1.452236-1.317920 \quad-0.893551$

$\mathrm{H} \quad 1.452236-1.317920 \quad 0.893551$

$\mathrm{H} \quad 0.111505-2.068963 \quad 0.000000$

$\mathrm{E}=-1430.9722128 \mathrm{NIMAG}=1$

INT-1a $\operatorname{MP}(\mathrm{FC}) / 6-31+\mathrm{G}$ *

S $0.000000 \quad 0.3250920 .000000$

O $\quad 1.117187 \quad 1.3214230 .000000$

Cl $-0.501545-0.101592 \quad 2.469156$

Cl $-0.501545-0.101592-2.469156$

C $\quad 0.850256-1.2709830 .000000$

$\mathrm{H} \quad 1.454841-1.312489-0.905339$

$\mathrm{H} \quad 1.454841-1.312489 \quad 0.905339$

$\mathrm{H} \quad 0.103807 \quad-2.067848 \quad 0.000000$

$E=-1431.7079566 \mathrm{NIMAG}=0$

INT-1a B3LYP/6-31+G*

S $\quad 0.000000 \quad 0.3202460 .000000$

o $\quad 1.0549121 .3764420 .000000$

Cl $-0.511300-0.1150942 .496592$

Cl $-0.511300-0.115094-2.496592$

C $\quad 0.939197-1.2640890 .000000$

$\mathrm{H} \quad 1.543957 \quad-1.275017-0.907365$

$\mathrm{H} \quad 1.543957-1.275017 \quad 0.907365$

$\mathrm{H} \quad 0.221817-2.087701 \quad 0.000000$

$E=-1433.8069341 \mathrm{NIMAG}=0$

INT-1a B3LYP/aug-cC-pVDZ

S $\quad 0.000000 \quad 0.315880 \quad 0.000000$

O $\quad 1.100888 \quad 1.3497510 .000000$

$\mathrm{Cl}-0.510229-0.098334 \quad 2.481799$

$\mathrm{Cl}-0.510229-0.098334-2.481799$

C $\quad 0.899027-1.296896 \quad 0.000000$

$\mathrm{H} \quad 1.499107-1.316493 \quad-0.913494$

$\mathrm{H} \quad 1.499107 \quad-1.316493 \quad 0.913494$

$\mathrm{H} \quad 0.148294 \quad-2.094367 \quad 0.000000$

$\mathrm{E}=-1433.8881909 \mathrm{NIMAG}=0$

R-1b ( $\mathrm{HSOCl}) \mathrm{HF} / 6-31+\mathrm{G}^{*}$

S $\quad 0.638028-0.443263-0.108271$

$\begin{array}{lllll}0 & 1.515747 & 0.699996 & 0.058828\end{array}$

$\begin{array}{lllll}C 1 & -1.346812 & 0.147994 & 0.009752\end{array}$

$\mathrm{H} \quad 0.561374-1.0236651 .095916$

$\mathrm{E}=-932.3542392 \mathrm{NIMAG}=0$

R-1b ( $\mathrm{HSOCl}) \quad \mathrm{MP}(\mathrm{FC}) / 6-31+\mathrm{G}$ *

S $\quad 0.660289-0.449587-0.109813$

$\begin{array}{lllll}0 & 1.589271 & 0.702320 & 0.059688\end{array}$

$\begin{array}{llll}\text { Cl } & -1.401145 & 0.152409 & 0.008802\end{array}$

$\mathrm{H} \quad 0.540683-1.016128 \quad 1.129867$

$\mathrm{E}=-932.8079524 \mathrm{NIMAG}=0$
R-1b (HSOCl) B3LYP/6-31+G*

S $\quad 0.673996-0.448879-0.111189$

$\begin{array}{lllll}0 & 1.593800 & 0.707166 & 0.060782\end{array}$

$\mathrm{Cl}-1.4172120 .149693 \quad 0.008863$

$\mathrm{H} \quad 0.558268-1.0200421 .142097$

$\mathrm{E}=-934.1559735 \mathrm{NIMAG}=0$

R-1b (HSOCl) B3LYP/aug-cC-pVDZ

S $\quad 0.675250-0.458871-0.110460$

O $\quad 1.598354 \quad 0.718404 \quad 0.058216$

Cl $-1.4198890 .152540 \quad 0.007875$

$\mathrm{H} \quad 0.547276 \quad-0.998479 \quad 1.167767$

$\mathrm{E}=-934.2166423 \mathrm{NIMAG}=0$

IDC-1b $\mathrm{HF} / 6-31+\mathrm{G}$ *

S $\quad 0.628218 \quad 0.630388 \quad 0.394223$

O $\quad 1.188997 \quad 1.690569-0.446766$

Cl $1.574273-1.192794-0.093233$

Cl $-2.692644-0.210634-0.059037$

$\mathrm{H} \quad-0.551167 \quad 0.247513-0.144841$

$\mathrm{E}=-1391.9269112 \mathrm{NIMAG}=0$

IDC-1b MP ( FC) / 6-31+G*

S $\quad 0.579421 \quad 0.685724 \quad 0.445949$

o $\quad 0.940491 \quad 1.789348-0.524212$

Cl $1.582010-1.203418-0.110834$

Cl $-2.527510-0.295131-0.066047$

$\mathrm{H} \quad-0.721165 \quad 0.188973 \quad 0.065483$

$\mathrm{E}=-1392.5202757 \mathrm{NIMAG}=0$

IDC-1b B3LYP/6-31+G*

$\begin{array}{llll}\text { S } & 0.533002 & 0.708707 & 0.457101\end{array}$

$\begin{array}{lllll}0 & 0.808177 & 1.820014 & -0.540467\end{array}$

Cl $1.660847-1.168925-0.113508$

Cl $-2.490537-0.361205-0.068260$

$\mathrm{H} \quad-0.888723 \quad 0.112788 \quad 0.100178$

$\mathrm{E}=-1394.4748436 \mathrm{NIMAG}=0$

IDC-1b B3LYP/aug-cC-pVDZ

$\begin{array}{llll}\text { S } & 0.527342 & 0.711080 & 0.471976\end{array}$

O $\quad 0.772153 \quad 1.831181-0.557076$

Cl $1.675588-1.160046-0.118568$

$\mathrm{Cl}-2.477995-0.375101-0.069814$

$\mathrm{H} \quad-0.973778 \quad 0.070756 \quad 0.107482$

$\mathrm{E}=-1394.5619927 \mathrm{NIMAG}=0$

TS-1b $\mathrm{HF} / 6-31+\mathrm{G}$ *

$\mathrm{S} \quad-0.381214 \quad 0.469665 \quad-0.256084$

$\begin{array}{lllll}0 & -0.813331 & 1.775038 & 0.219659\end{array}$

Cl $-2.008756-0.912437 \quad 0.059039$

Cl $2.731799-0.359990 \quad 0.035606$

$\mathrm{H} \quad 0.314356-0.083668 \quad 0.731121$

$\mathrm{E}=-1391.9260139 \mathrm{NIMAG}=1$ 
TS-1b MP (FC) / 6-31+G*

$\begin{array}{lrrr}\mathrm{S} & 0.344242 & 0.529600 & 0.309098 \\ \mathrm{O} & 0.716687 & 1.858529 & -0.283887 \\ \mathrm{Cl} & 2.001616 & -0.949883 & -0.074393 \\ \mathrm{Cl} & -2.634722 & -0.417588 & -0.048614 \\ \mathrm{H} & -0.478576 & -0.094813 & -0.583350 \\ \mathrm{E}= & -1392.5174599 & \mathrm{NIMAG}=1\end{array}$

TS-1b B3LYP/6-31+G*

$\begin{array}{llll}\mathrm{S} & 0.315673 & 0.521772 & 0.328130\end{array}$

$\begin{array}{llll}0 & 0.590738 & 1.855337 & -0.303017\end{array}$

Cl $2.093993-0.897735-0.082090$

$\mathrm{Cl}-2.636817-0.456449-0.053865$

$\mathrm{H} \quad-0.548668 \quad-0.169914 \quad-0.514724$

$\mathrm{E}=-1394.47070497 \mathrm{NIMAG}=1$

TS-1b B3LYP/aug-cC-pVDZ

S $\quad 0.272429 \quad 0.474650 \quad 0.328876$

o $\quad 0.479946 \quad 1.845747-0.289939$

Cl $2.150311-0.826903-0.084943$

$\mathrm{Cl}-2.600083-0.473572-0.056222$

$\mathrm{H} \quad-0.552308-0.252296-0.542706$

$\mathrm{E}=-1394.5559843 \mathrm{NIMAG}=1$

INT-1b $\mathrm{HF} / 6-31+\mathrm{G}^{*}$

$\begin{array}{lrrr}\mathrm{S} & 0.000000 & 0.232055 & 0.000000 \\ \mathrm{O} & 1.024510 & 1.244763 & 0.000000 \\ \mathrm{Cl} & -0.259798 & -0.374690 & 2.424562 \\ \mathrm{Cl} & -0.259798 & -0.374690 & -2.424562 \\ \mathrm{H} & 0.637069 & -0.931527 & 0.000000 \\ \mathrm{E}= & -1391.9269611 & \mathrm{NIMAG}= & 0\end{array}$

INT-1b MP (FC) / 6-31+G*

S $\quad 0.000000 \quad 0.253765 \quad 0.000000$

$\begin{array}{llll}0 & 0.980594 & 1.384205 & 0.000000\end{array}$

Cl $-0.252690-0.419325 \quad 2.421869$

$\mathrm{Cl}-0.252690-0.419325-2.421869$

$\mathrm{H} \quad 0.746712 \quad-0.876833 \quad 0.000000$

$\mathrm{E}=-1392.5245753 \mathrm{NIMAG}=0$

INT-1b B3LYP/6-31+G*

$\begin{array}{lrrr}\mathrm{S} & 0.000000 & 0.247728 & 0.000000 \\ \mathrm{O} & 0.929593 & 1.416723 & 0.000000 \\ \mathrm{Cl} & -0.242190 & -0.424489 & 2.442392 \\ \mathrm{Cl} & -0.242190 & -0.424489 & -2.442392 \\ \mathrm{H} & 0.797703 & -0.864794 & 0.000000 \\ \mathrm{E}= & -1394.4806231 & \mathrm{NIMAG}= & 0\end{array}$

INT-1b B3LYP/aug-cC-pVDZ

S $0.000000 \quad 0.2406920 .000000$

$\begin{array}{llll}0 & 1.028687 & 1.348905 & 0.000000\end{array}$

Cl $-0.263338-0.403221 \quad 2.428156$

Cl $-0.263338-0.403221-2.428156$

$\mathrm{H} \quad 0.724002 \quad-0.932796 \quad 0.000000$

$\mathrm{E}=-1394.5650745 \mathrm{NIMAG}=0$
R-1c (PhSOCl) HF/6-31+G*

$\begin{array}{lrrr}\mathrm{S} & -0.390864 & 1.263050 & 0.810098 \\ \mathrm{O} & -0.573863 & 2.339863 & -0.149640 \\ \mathrm{C} 1 & 1.625840 & 1.275197 & 1.394949 \\ \mathrm{C} & -0.364911 & -0.254446 & -0.124980 \\ \mathrm{C} & -0.423671 & -1.461484 & 0.558620 \\ \mathrm{C} & -0.459131 & -2.643315 & -0.161384 \\ \mathrm{C} & -0.443078 & -2.607003 & -1.550315 \\ \mathrm{C} & -0.394730 & -1.393990 & -2.221900 \\ \mathrm{C} & -0.359040 & -0.204268 & -1.509254 \\ \mathrm{H} & -0.434393 & -1.481954 & 1.634302 \\ \mathrm{H} & -0.501346 & -3.584556 & 0.355294 \\ \mathrm{H} & -0.472640 & -3.525682 & -2.107849 \\ \mathrm{H} & -0.387792 & -1.372019 & -3.296289 \\ \mathrm{H} & -0.331021 & 0.745191 & -2.008770 \\ \mathrm{E} & =-1161.91773051 \mathrm{NIMAG}=0\end{array}$

R-1c (PhSOCl) MP2 (FC) /6-31+G* $\begin{array}{llll}\mathrm{Cl} & -0.420592 & 1.279677 & 0.809011\end{array}$

$\begin{array}{llll}0 & -0.630709 & 2.372725 & -0.183912\end{array}$

S $\quad 1.666790 \quad 1.239116 \quad 1.424733$

C $\quad-0.334926-0.246411-0.127847$

$\begin{array}{llll}\text { C } & -0.422161 & -1.456803 & 0.568809\end{array}$

C $\quad-0.453727-2.648942-0.160075$

C $\quad-0.450881-2.616269-1.560599$

C $\quad-0.388328-1.391693-2.237781$

C $-0.355688-0.190423-1.522706$

$\mathrm{H} \quad-0.417845-1.474741 \quad 1.658211$

$\begin{array}{llll}\mathrm{H} & -0.509908 & -3.600526 & 0.363918\end{array}$

$\mathrm{H} \quad-0.483159-3.546509-2.123681$

$\mathrm{H} \quad-0.393979-1.370639-3.325431$

$\begin{array}{lllll}\mathrm{H} & -0.321131 & 0.774069 & -2.025158\end{array}$

$\mathrm{E}=-1163.12777532 \mathrm{NIMAG}=0$

R-1c (PhSOCl) B3LYP/6-31+G*

$\begin{array}{llll}\mathrm{S} & -0.425165 & 1.291868 & 0.793369\end{array}$

$\begin{array}{llll}0 & -0.575584 & 2.385482 & -0.206589\end{array}$

$\begin{array}{llll}\mathrm{Cl} & 1.674746 & 1.247204 & 1.493146\end{array}$

C $\quad-0.375066-0.253549-0.147991$

$\begin{array}{llll}\text { C } & -0.420605 & -1.465456 & 0.549176\end{array}$

C $\quad-0.455940-2.657783-0.175350$

$\begin{array}{lllll}\text { C } & -0.448540 & -2.626740 & -1.574857\end{array}$

C $\quad-0.409956-1.405175-2.255883$

C $\quad-0.376874-0.204491-1.542903$

$\mathrm{H} \quad-0.416083-1.481958 \quad 1.636429$

$\mathrm{H} \quad-0.487721-3.608079 \quad 0.350192$

$\mathrm{H} \quad-0.475798-3.557455-2.135133$

$\mathrm{H} \quad-0.407505-1.386643-3.342252$

$\mathrm{H} \quad-0.3543820 .757093-2.047053$

$\mathrm{E}=-1165.21972599 \mathrm{NIMAG}=0$ 
R-1C (PhSOCl) B3LYP/aug-cc-pVDZ

$\begin{array}{lrrr}\mathrm{S} & -0.427386 & 1.291273 & 0.807172 \\ \mathrm{O} & -0.590009 & 2.396970 & -0.203012 \\ \mathrm{C} 1 & 1.688344 & 1.245469 & 1.468951 \\ \mathrm{C} & -0.380637 & -0.257519 & -0.144024 \\ \mathrm{C} & -0.427665 & -1.468088 & 0.554323 \\ \mathrm{C} & -0.460358 & -2.660253 & -0.172024 \\ \mathrm{C} & -0.447709 & -2.627220 & -1.571886 \\ \mathrm{C} & -0.406453 & -1.404620 & -2.252165 \\ \mathrm{C} & -0.376400 & -0.203779 & -1.538158 \\ \mathrm{H} & -0.426629 & -1.482595 & 1.644821 \\ \mathrm{H} & -0.494758 & -3.614542 & 0.353578 \\ \mathrm{H} & -0.472706 & -3.560377 & -2.135235 \\ \mathrm{H} & -0.400243 & -1.385403 & -3.342118 \\ \mathrm{H} & -0.353931 & 0.762694 & -2.040281 \\ \mathrm{E} & =-1165.303068 & -1.04 M A G & =\end{array}$

IDC-1C $\mathrm{HF} / 6-31+\mathrm{G}$ *

\begin{tabular}{|c|c|c|c|}
\hline S & 0.087311 & 1.149465 & 0.616207 \\
\hline O & 0.018721 & 2.207344 & -0.357730 \\
\hline $\mathrm{Cl}$ & 2.454062 & 0.949461 & 0.989061 \\
\hline $\mathrm{Cl}$ & -2.576301 & 0.787190 & 1.156713 \\
\hline $\mathrm{C}$ & 0.049656 & -0.374337 & -0.320233 \\
\hline C & -0.001128 & -1.579748 & 0.361104 \\
\hline $\mathrm{C}$ & -0.031084 & -2.761789 & -0.35696 \\
\hline $\mathrm{C}$ & -0.014707 & -2.730607 & -1.746404 \\
\hline $\mathrm{C}$ & 0.029216 & -1.517605 & -2.416288 \\
\hline $\mathrm{C}$ & 0.060613 & -0.328338 & -1.70103 \\
\hline $\mathrm{H}$ & -0.03 & -1.592524 & 1.4 \\
\hline $\mathrm{H}$ & -0.075609 & -3.702452 & 0.1624 \\
\hline $\mathrm{H}$ & -0.041960 & -3.651155 & $-2 \cdot 3$ \\
\hline $\mathrm{H}$ & 0.03 & -1.494124 & -3.49177 \\
\hline $\mathrm{H}$ & 0.08 & 0.621 & -2.19890 \\
\hline & -162 & 085 NIMAG & $=0$ \\
\hline
\end{tabular}

IDC-1C MP2 (FC) /6-31+G*

$\mathrm{S} \quad-1.440384-0.831599-0.695376$

$\begin{array}{lllll}0 & -2.469040 & -0.234062 & -1.604307\end{array}$

$\mathrm{Cl}-2.416196-1.156648 \quad 1.266952$

Cl $3.687249-1.721670-0.188336$

$\begin{array}{llll}\text { C } & -0.391911 & 0.496705 & -0.159384\end{array}$

C $\quad 0.967893 \quad 0.202362 \quad-0.036117$

$\begin{array}{llll}\text { C } & 1.844253 & 1.248807 & 0.281085\end{array}$

C $\quad 1.358237 \quad 2.551352 \quad 0.431623$

$\begin{array}{lrrr}\text { C } & -0.012175 & 0.823613 & 0.278819\end{array}$

C $\quad-0.901084 \quad 1.796382-0.040226$

$\mathrm{H} \quad 1.400982-0.794217-0.160072$

$\begin{array}{llll}\mathrm{H} & 2.900144 & 1.001021 & 0.369088\end{array}$

$\mathrm{H} \quad 2.044517 \quad 3.361479 \quad 0.674110$

$\mathrm{H} \quad-0.382379 \quad 3.842095 \quad 0.386857$

$\mathrm{H} \quad-1.963971 \quad 1.983777-0.180795$

$\mathrm{E}=-1622824180 \mathrm{NIMAG}=0$
IDC-1C B3LYP/6-31+G*

$\begin{array}{lrrr}\mathrm{S} & -1.525263 & -0.795598 & -0.743639 \\ \mathrm{O} & -2.656016 & -0.159468 & -1.488059 \\ \mathrm{Cl} & -2.344707 & -1.357935 & 1.278633 \\ \mathrm{Cl} & 3.828429 & -1.591765 & -0.146889 \\ \mathrm{C} & -0.427873 & 0.521332 & -0.227060 \\ \mathrm{C} & 0.918054 & 0.194072 & -0.038789 \\ \mathrm{C} & 1.814022 & 1.217594 & 0.293940 \\ \mathrm{C} & 1.353895 & 2.527520 & 0.434323 \\ \mathrm{C} & -0.002698 & 2.837832 & 0.233235 \\ \mathrm{C} & -0.905943 & 1.834040 & -0.104421 \\ \mathrm{H} & 1.328702 & -0.811304 & -0.151409 \\ \mathrm{H} & 2.857616 & 0.936107 & 0.415910 \\ \mathrm{H} & 2.051990 & 3.320502 & 0.694183 \\ \mathrm{H} & -0.349724 & 3.863553 & 0.337590 \\ \mathrm{H} & -1.956287 & 2.047002 & -0.280598 \\ \mathrm{E} & = & -1625.514819 & \text { NIMAG }\end{array}$

IDC-1c B3LYP/aug-cC-pVDZ

S $\quad-1.536984-0.793360-0.755970$

O $\quad-2.703670-0.133150-1.458001$

Cl $-2.287272-1.398033 \quad 1.277495$

Cl $3.807205-1.564604-0.144638$

C $\quad-0.428404 \quad 0.525857-0.235431$

C $\quad 0.912925 \quad 0.189910-0.032030$

$\begin{array}{llll}\text { C } & 1.810166 & 1.211461 & 0.305637\end{array}$

C $\quad 1.353069 \quad 2.524032 \quad 0.439459$

C $\quad-0.000562 \quad 2.839730 \quad 0.224696$

C $\quad-0.905453 \quad 1.838913-0.120761$

$\mathrm{H} \quad 1.324509-0.819971-0.140764$

$\begin{array}{llll}\mathrm{H} & 2.855167 & 0.922356 & 0.435746\end{array}$

$\begin{array}{llll}\mathrm{H} & 2.053912 & 3.317706 & 0.704867\end{array}$

$\mathrm{H} \quad-0.345489 \quad 3.870512 \quad 0.322616$

$\mathrm{H} \quad-1.956323 \quad 2.053768-0.310944$

$\mathrm{E}=-1625.624948 \mathrm{NIMAG}=0$

TS-1C $\mathrm{HF} / 6-31+\mathrm{G}^{*}$

\begin{tabular}{|c|c|c|c|}
\hline S & 0.000000 & 1.142697 & 0.625535 \\
\hline 0 & 0.000000 & 2.200956 & -0.348538 \\
\hline $\mathrm{Cl}$ & 2.506140 & 0.878760 & 1.061072 \\
\hline & -2.506140 & 0.878760 & 1.061072 \\
\hline & 0.000000 & -0.379546 & -0.313596 \\
\hline & 0.000000 & -1.587578 & 0.365222 \\
\hline & 0.000000 & -2.768625 & -0.3 \\
\hline & 0.000000 & -2.734579 & -1.74429 \\
\hline & 0.00 & -1.519529 & -2.4 \\
\hline & 0.000000 & -0.331087 & -1.69 \\
\hline & 0.000000 & -1.603624 & 1.4 \\
\hline & 0.00 & -3.711155 & 0.163278 \\
\hline & 0.00 & -3.654505 & -2.302874 \\
\hline & & -1.493668 & \\
\hline & 0.0 & 0.619986 & -2.190395 \\
\hline & & $058 \mathrm{~N}$ & $=1$ \\
\hline
\end{tabular}


TS-1c $\operatorname{MP} 2(\mathrm{FC}) / 6-31+\mathrm{G}^{*}$

$\begin{array}{lrrr}\mathrm{S} & 0.759281 & -1.253040 & 0.650603 \\ \mathrm{O} & 1.772899 & -1.226012 & 1.752341 \\ \mathrm{C} 1 & 1.844507 & -1.771269 & -1.214842 \\ \mathrm{Cl} & -3.772306 & -0.864812 & -0.132476 \\ \mathrm{C} & 0.465652 & 0.444490 & 0.200311 \\ \mathrm{C} & -0.858002 & 0.787237 & -0.084774 \\ \mathrm{C} & -1.137789 & 2.135956 & -0.343567 \\ \mathrm{C} & -0.126340 & 3.099983 & -0.280034 \\ \mathrm{C} & 1.190045 & 2.725537 & 0.037017 \\ \mathrm{C} & 1.494446 & 1.389313 & 0.300394 \\ \mathrm{H} & -1.680824 & 0.059429 & -0.114670 \\ \mathrm{H} & -2.165794 & 2.414344 & -0.566452 \\ \mathrm{H} & -0.356868 & 4.145647 & -0.479423 \\ \mathrm{H} & 1.973208 & 3.479807 & 0.097286 \\ \mathrm{H} & 2.503098 & 1.075782 & 0.563208 \\ \mathrm{E} & -1622.824026 \mathrm{NIMAG} & =1\end{array}$

TS-1c B3LYP/6-31+G*

$\begin{array}{lrrr}\mathrm{S} & 0.717657 & -1.207901 & 0.630458 \\ \mathrm{O} & 1.807894 & -1.218352 & 1.654398 \\ \mathrm{C} 1 & 1.694426 & -1.796180 & -1.319120 \\ \mathrm{Cl} & -3.608713 & -1.083535 & 0.073613 \\ \mathrm{C} & 0.433725 & 0.523073 & 0.219426 \\ \mathrm{C} & -0.862014 & 0.883668 & -0.158902 \\ \mathrm{C} & -1.111077 & 2.236109 & -0.428021 \\ \mathrm{C} & -0.090349 & 3.182068 & -0.317857 \\ \mathrm{C} & 1.200762 & 2.793903 & 0.076004 \\ \mathrm{C} & 1.470129 & 1.455645 & 0.352778 \\ \mathrm{H} & -1.683819 & 0.153622 & -0.206965 \\ \mathrm{H} & -2.117461 & 2.533174 & -0.710958 \\ \mathrm{H} & -0.295309 & 4.229564 & -0.529939 \\ \mathrm{H} & 1.990653 & 3.536092 & 0.167448 \\ \mathrm{H} & 2.456110 & 1.129136 & 0.670946 \\ \mathrm{E} & -1625.514068 & -1.61 M A G & =\end{array}$

TS-1c B3LYP/aug-cC-pVDZ

$\begin{array}{lrrr}\mathrm{S} & 0.710618 & -1.209583 & 0.635083 \\ \mathrm{O} & 1.842474 & -1.211890 & 1.639762 \\ \mathrm{C} 1 & 1.629956 & -1.798366 & -1.336723 \\ \mathrm{C} 1 & -3.546414 & -1.095133 & 0.099813 \\ \mathrm{C} & 0.424417 & 0.531164 & 0.221423 \\ \mathrm{C} & -0.866599 & 0.888385 & -0.174719 \\ \mathrm{C} & -1.109337 & 2.242476 & -0.445404 \\ \mathrm{C} & -0.087756 & 3.187286 & -0.324441 \\ \mathrm{C} & 1.198458 & 2.797809 & 0.084721 \\ \mathrm{C} & 1.462685 & 1.458635 & 0.366462 \\ \mathrm{H} & -1.692411 & 0.153117 & -0.225572 \\ \mathrm{H} & -2.115006 & 2.543936 & -0.740645 \\ \mathrm{H} & -0.289457 & 4.238406 & -0.540030 \\ \mathrm{H} & 1.990539 & 3.541526 & 0.185900 \\ \mathrm{H} & 2.445223 & 1.126419 & 0.700147 \\ \mathrm{E} & -1625.624172 & -1.71 M A G & =\end{array}$

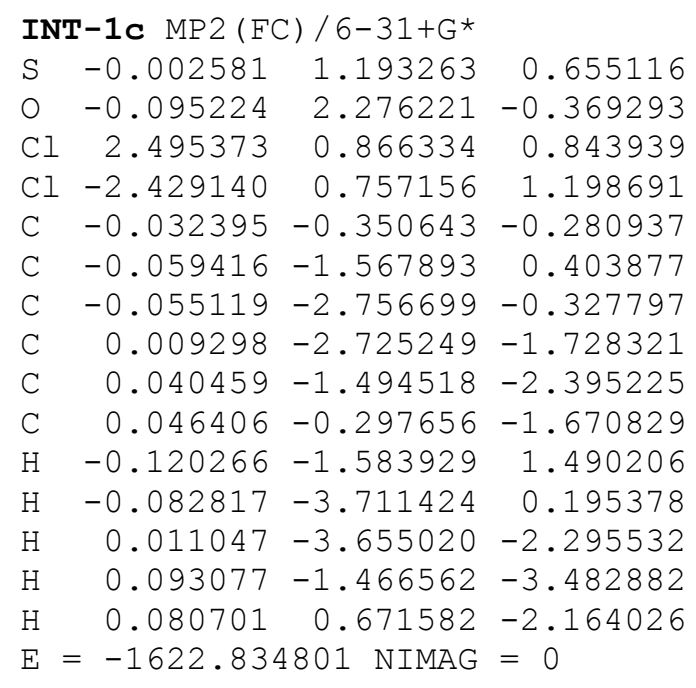

INT-1C B3L/6-31+G*

$\begin{array}{lrrr}\mathrm{S} & 0.000000 & 1.181677 & 0.620463 \\ \mathrm{O} & 0.000000 & 2.255146 & -0.414187 \\ \mathrm{Cl} & 2.503369 & 0.867422 & 1.096536 \\ \mathrm{Cl} & -2.503369 & 0.867422 & 1.096536 \\ \mathrm{C} & 0.000000 & -0.387257 & -0.321078 \\ \mathrm{C} & 0.000000 & -1.604059 & 0.362663 \\ \mathrm{C} & 0.000000 & -2.793673 & -0.366352 \\ \mathrm{C} & 0.000000 & -2.761223 & -1.766767 \\ \mathrm{C} & 0.000000 & -1.533678 & -2.436473 \\ \mathrm{C} & 0.000000 & -0.337645 & -1.712120 \\ \mathrm{H} & 0.000000 & -1.621181 & 1.448797 \\ \mathrm{H} & 0.000000 & -3.747097 & 0.157410 \\ \mathrm{H} & 0.000000 & -3.690887 & -2.332170 \\ \mathrm{H} & 0.000000 & -1.506133 & -3.524258 \\ \mathrm{H} & 0.000000 & 0.630183 & -2.205162 \\ \mathrm{E} & = & -1625.52836874 & \mathrm{NIMAG}\end{array}$

$\mathrm{CN}^{-} \mathrm{HF} / 6-31+\mathrm{G}$ *

$\begin{array}{llll}C & 0.000000 & 0.000000 & -0.625453 \\ N & 0.000000 & 0.000000 & 0.536102 \\ E= & -92.3148718 \quad \mathrm{NIMAG}= & 0\end{array}$

$\mathrm{CN}^{-} \mathrm{MP}(\mathrm{FC}) / 6-31+\mathrm{G}^{*}$

C $\quad 0.000000 \quad 0.000000-0.647147$

$\mathrm{N} \quad 0.000000 \quad 0.000000 \quad 0.554698$

$E=-92.6034029 \mathrm{NIMAG}=0$
$\mathrm{CN}^{-}$B3LYP/6-31+G*
C $\quad 0.000000 \quad 0.000000-0.637273$
$\mathrm{N} \quad 0.000000 \quad 0.000000 \quad 0.546234$
$\mathrm{E}=-92.8654293 \mathrm{NIMAG}=0$
$\mathrm{CN}^{-}$B3LYP/aug-cC-pVDZ
C $\quad 0.000000 \quad 0.000000-0.637273$
$\mathrm{N} \quad 0.000000 \quad 0.000000 \quad 0.546234$
$\mathrm{E}=-92.8736605 \mathrm{NIMAG}=0$




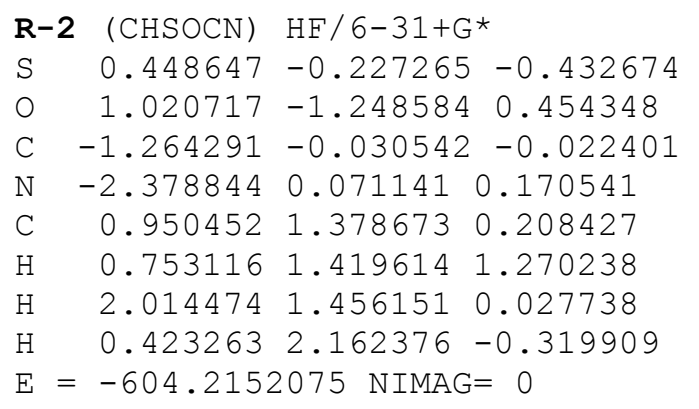

R-2 ( $\mathrm{CHSOCN}) \mathrm{MP}(\mathrm{FC}) / 6-31+\mathrm{G}$ *

S $\quad 0.462194 \quad-0.220181-0.438871$

o $\quad 1.049326-1.274403 \quad 0.454671$

C $\quad-1.262293 \quad 0.008690 \quad-0.025049$

$\begin{array}{lllll}\mathrm{N} & -2.433251 & 0.026527 & 0.171108\end{array}$

C $\quad 0.943930 \quad 1.396568 \quad 0.219308$

$\mathrm{H} \quad 0.754187 \quad 1.416915 \quad 1.294566$

H $\quad 2.013890 \quad 1.502355 \quad 0.024735$

$\mathrm{H} \quad 0.385145 \quad 2.181608-0.298042$

$\mathrm{E}=-604.9524335 \mathrm{NIMAG}=0$

\section{R-2 (CHSOCN) B3LYP/6-31+G* \\ S $\quad 0.457238-0.232416-0.438583$ \\ O $1.048402-1.2790920 .457606$ \\ C $\quad-1.2808990 .003667-0.014838$ \\ $\mathrm{N} \quad-2.429803 \quad 0.044844 \quad 0.168516$ \\ C $\quad 0.962346 \quad 1.412883 \quad 0.213298$ \\ $\mathrm{H} \quad 0.760373 \quad 1.451582 \quad 1.285957$ \\ H $\quad 2.036259 \quad 1.4901230 .024361$ \\ $\mathrm{H} \quad 0.4202812 .196486-0.324208$ \\ $\mathrm{E}=-606.1015844 \mathrm{NIMAG}=0$}

\section{R-2 (CHSOCN) B3LYP/aug-CC-pVDZ \\ S $\quad 0.455384-0.226707-0.448736$ \\ O $1.049920-1.2870520 .464156$ \\ C $\quad-1.285587-0.004806-0.014428$ \\ $\mathrm{N} \quad-2.432972 \quad 0.050692 \quad 0.172913$ \\ C $\quad 0.970073 \quad 1.414892 \quad 0.222271$ \\ H $\quad 0.763488 \quad 1.431527 \quad 1.297112$ \\ $\mathrm{H} \quad 2.046840 \quad 1.474155 \quad 0.027022$ \\ $\mathrm{H} \quad 0.428065 \quad 2.202674-0.315049$ \\ $\mathrm{E}=-606.1390317 \mathrm{NIMAG}=0$}

IDC-2 $\mathrm{HF} / 6-31+\mathrm{G}$ *

$\mathrm{S} \quad-0.4848030 .384306-0.296045$

O $\quad-1.078760 \quad 1.735419-0.234165$

C $0.103434 \quad 0.007599 \quad 1.359093$

H $\quad 0.982084 \quad 0.617026 \quad 1.497251$

$\mathrm{H} \quad-0.680068 \quad 0.247364 \quad 2.064982$

$\mathrm{H} \quad 0.397854 \quad-1.030821 \quad 1.404374$

C $\quad-1.868177-0.756015-0.213026$

C $\quad 2.919493-0.166910-0.198937$

$\mathrm{N} \quad-2.745191-1.479489-0.242721$

$\mathrm{N} \quad 3.996413-0.573921-0.334328$

$\mathrm{E}=-696.5553356 \mathrm{NIMAG}=0$
IDC-2 $\mathrm{MP}(\mathrm{FC}) / 6-31+\mathrm{G}$ *

S $\quad-0.286400 \quad 0.279695-0.172472$

$\begin{array}{lllll}0 & -0.489525 & 1.708072 & 0.260447\end{array}$

$\begin{array}{llll}\mathrm{C} & -1.981200 & -0.416562 & -0.373133\end{array}$

C $\quad 2.630672-0.158937-0.200638$

$\mathrm{N} \quad-3.088874-0.737621-0.661622$

$\mathrm{N} \quad 3.743433-0.401220-0.577283$

C $\quad-0.020976-0.687430 \quad 1.332243$

$\mathrm{H} \quad 0.976504-0.403467 \quad 1.667001$

$\mathrm{H} \quad-0.801989-0.435705 \quad 2.053196$

$\mathrm{H} \quad-0.028809-1.751070 \quad 1.077279$

$E=-697.5849834 \mathrm{NIMAG}=0$

\section{IDC-2 B3LYP/6-31+G*}

S $\quad 0.663606 \quad 0.400650-0.319782$

$\begin{array}{lllll}\mathrm{O} & 0.955983 & 1.760691 & 0.257031\end{array}$

$\begin{array}{lllll}\mathrm{C} & 2.197258 & -0.576485 & -0.204237\end{array}$

N $\quad 3.224718-1.123826-0.262901$

C $\quad-0.249255-0.580833 \quad 0.919850$

$\begin{array}{lllll}\mathrm{H} & 0.148456 & -0.334644 & 1.908277\end{array}$

$\mathrm{H} \quad-1.318102-0.314338 \quad 0.784380$

$\mathrm{H} \quad-0.118181-1.642294 \quad 0.685930$

C $\quad-3.341970-0.194572-0.012679$

$\mathrm{N} \quad-4.455282-0.318077-0.385089$

$E=-698.9933367 \mathrm{NIMAG}=0$

\section{IDC-2 B3LYP/aug-cC-pVDZ}

S $\quad 0.653727 \quad 0.393103-0.329813$

$\begin{array}{lllll}0 & 0.962091 & 1.770607 & 0.253424\end{array}$

C $\quad 2.192792-0.579281-0.203115$

$\mathrm{N} \quad 3.216017-1.135659-0.243731$

C $\quad-0.271134-0.567292 \quad 0.925609$

$\mathrm{H} \quad 0.142474-0.310092 \quad 1.907184$

$\mathrm{H} \quad-1.338443-0.279004 \quad 0.772372$

$\mathrm{H} \quad-0.147021-1.632241 \quad 0.689612$

$\begin{array}{lllll}\mathrm{C} & -3.313783 & -0.165975 & -0.019982\end{array}$

$\mathrm{N}-4.424678-0.344038-0.375502$

$\mathrm{E}=-699.0400429 \mathrm{NIMAG}=0$

TS-2 B3LYP/6-31+G*

S $\quad 0.573534 \quad 0.372930 \quad-0.282157$

$\begin{array}{lllll}0 & 0.875543 & 1.765808 & 0.202990\end{array}$

C $\quad 2.144073-0.556960-0.251595$

$\begin{array}{lllll}\mathrm{N} & 3.181367 & -1.074477 & -0.374318\end{array}$

C $\quad-0.181806-0.5665051 .091978$

$\mathrm{H} \quad 0.301646-0.257036 \quad 2.022536$

$\mathrm{H} \quad-1.257151-0.320745 \quad 1.025157$

$\mathrm{H} \quad-0.047964-1.635725 \quad 0.900153$

C $-3.198826-0.189192-0.041581$

$\mathrm{N}-4.289660-0.354651-0.461406$

$\mathrm{E}=-698.9933107 \mathrm{NIMAG}=1$ 
TS-2 B3LYP/aug-cC-pVDZ

$\begin{array}{lrll}\mathrm{S} & 0.578899 & 0.370245 & -0.297982 \\ \mathrm{O} & 0.894106 & 1.775081 & 0.210703 \\ \mathrm{C} & 2.150043 & -0.561444 & -0.241864 \\ \mathrm{~N} & 3.183376 & -1.092326 & -0.335231 \\ \mathrm{C} & -0.216418 & -0.559491 & 1.067299 \\ \mathrm{H} & 0.264945 & -0.249816 & 2.001521 \\ \mathrm{H} & -1.290237 & -0.290719 & 0.965241 \\ \mathrm{H} & -0.087747 & -1.630668 & 0.865641 \\ \mathrm{C} & -3.190728 & -0.165313 & -0.058240 \\ \mathrm{~N} & -4.291887 & -0.369942 & -0.429553 \\ \mathrm{E} & =-699.0400264 & \text { NIMAG }= & 1\end{array}$

INT-2 B3LYP/6-31+G*

S $\quad-0.099408-0.254787$ -

0.095824

$\begin{array}{lllll}0 & -0.103046 & -1.351961 & 0.928875\end{array}$

C $\quad 2.283296-0.003227-0.328307$

C $\quad-2.088253 \quad 0.056300-0.357277$

$\mathrm{N} \quad 3.407061 \quad 0.105132 \quad-0.656870$

$\mathrm{N} \quad-3.191157 \quad 0.126815-0.742402$

C $\quad-0.035464 \quad 1.343087 \quad 0.835405$

$\mathrm{H} \quad-0.849416 \quad 1.347090 \quad 1.561973$

H $\quad 0.940061 \quad 1.380909 \quad 1.317324$

$\mathrm{H} \quad-0.144553 \quad 2.163697 \quad 0.118873$

$\mathrm{E}=-698.9974822 \mathrm{NIMAG}=0$

INT-2 B3LYP/aug-cC-pVDZ

S $\quad-0.003593-0.247871-0.102793$

$\begin{array}{lllll}0 & -0.003727 & -1.338008 & 0.955835\end{array}$

C $\quad 2.179463 \quad 0.016896-0.331814$

C $\quad-2.172174 \quad 0.019364-0.332999$

$\mathrm{N} \quad 3.289532 \quad 0.094326-0.702668$

$\mathrm{N} \quad-3.281493 \quad 0.094654 \quad-0.705879$

C $\quad-0.0015661 .3643250 .819286$

$\mathrm{H} \quad-0.904523 \quad 1.383090 \quad 1.433433$

$\mathrm{H} \quad 0.910031 \quad 1.3873561 .420196$

$\mathrm{H} \quad-0.008818 \quad 2.173179 \quad 0.077365$

$\mathrm{E}=-699.044905 \mathrm{NIMAG}=0$

TS2-2 $\mathrm{HF} / 6-31+\mathrm{G}^{*}$

S $\quad 0.000000 \quad 0.2726970 .000000$

o $\quad 1.426756 \quad 0.609074 \quad 0.000000$

C $\quad-0.268203 \quad 0.182910 \quad 2.130530$

C $\quad-0.268203 \quad 0.182910 \quad-2.130530$

$\mathrm{N} \quad-0.510514 \quad 0.244332 \quad 3.250977$

$\mathrm{N} \quad-0.510514 \quad 0.244332 \quad-3.250977$

C $-0.109159-1.537253 \quad 0.000000$

$\mathrm{H} \quad 0.381265-1.905352-0.886055$

$\mathrm{H} \quad 0.381265-1.905352 \quad 0.886055$

$\mathrm{H} \quad-1.155988-1.817087 \quad 0.000000$

$\mathrm{E}=-696.5402013 \mathrm{NIMAG}=1$
TS2-2 $\mathrm{MP}(\mathrm{FC}) / 6-31+\mathrm{G}$ *

S $0.000000 \quad 0.2506300 .000000$

O $\quad 1.3833150 .8414620 .000000$

$\begin{array}{lllll}\text { C } & -0.309717 & 0.084271 & 2.129246\end{array}$

C $\quad-0.309717 \quad 0.084271-2.129246$

$\mathrm{N} \quad-0.664063 \quad 0.235918 \quad 3.259458$

$\mathrm{N} \quad-0.664063 \quad 0.235918-3.259458$

C $\quad 0.204624-1.560806 \quad 0.000000$

$\mathrm{H} \quad 0.753938-1.834409-0.900188$

$\mathrm{H} \quad 0.753938-1.834409 \quad 0.900188$

$\mathrm{H} \quad-0.788651-2.0222120 .000000$

$\mathrm{E}=-697.5818143 \mathrm{NIMAG}=1$

\section{TS2-2 B3LYP/6-31+G*}

S $\quad 0.000000 \quad 0.2692430 .000000$

$\begin{array}{llll}0 & 1.372151 & 0.877928 & 0.000000\end{array}$

C $\quad-0.320358 \quad 0.106675 \quad 2.166769$

C $\quad-0.320358 \quad 0.106675 \quad-2.166769$

$\mathrm{N} \quad-0.669728 \quad 0.178718 \quad 3.284134$

$\mathrm{N} \quad-0.669728 \quad 0.178718 \quad-3.284134$

C $\quad 0.236142-1.568930 \quad 0.000000$

$\mathrm{H} \quad 0.789204-1.829662-0.901853$

$\mathrm{H} \quad 0.789204 \quad-1.829662 \quad 0.901853$

$\mathrm{H} \quad-0.751981-2.040561 \quad 0.000000$

$\mathrm{E}=-698.9973966 \mathrm{NIMAG}=1$

\section{TS2-2 B3LYP/aug-cc-pVDZ}

S $\quad 0.000000 \quad 0.2683370 .000000$

o $\quad 1.394336 \quad 0.872376 \quad 0.000000$

C $\quad-0.314323 \quad 0.109901 \quad 2.175624$

C $\quad-0.314323 \quad 0.109901 \quad-2.175624$

$\mathrm{N} \quad-0.688443 \quad 0.181330 \quad 3.284909$

$\mathrm{N}-0.688443 \quad 0.181330-3.284909$

C $\quad 0.238002-1.5736870 .000000$

$\mathrm{H} \quad 0.791775-1.824455-0.907296$

$\mathrm{H} \quad 0.791775-1.824455 \quad 0.907296$

$\mathrm{H} \quad-0.756172-2.038787 \quad 0.000000$

$\mathrm{E}=-699.0448932 \mathrm{NIMAG}=1$

$\mathrm{PH}^{-} \mathrm{HF} / 6-31+\mathrm{G}^{\star}$

P $\quad 0.000000 \quad 0.000000 \quad 0.114991$

$\mathrm{H} \quad 0.000000 \quad 1.037374 \quad-0.862434$

$\mathrm{H} \quad 0.000000-1.037374-0.862434$

$E=-341.852881601 \mathrm{NIMAG}=0$

\footnotetext{
$\mathrm{PH}^{-} \mathrm{MP}(\mathrm{FC}) / 6-31+\mathrm{G}$ *

P $\quad 0.000000 \quad 0.000000 \quad 0.116671$

$\mathrm{H} \quad 0.000000 \quad 1.034666-0.875035$

$\mathrm{H} \quad 0.000000-1.034666-0.875035$

$\mathrm{E}=-341.961234 \mathrm{NIMAG}=0$

$\mathrm{PH}^{-}$B3LYP/ 6-31+G*

P $0.000000 \quad 0.118058 \quad 0.000000$

H $\quad 1.039327-0.885460 \quad 0.000000$

$\mathrm{H} \quad-1.039327-0.885410 \quad 0.000000$

$\mathrm{E}=-342.5502396 \mathrm{NIMAG}=0$
} 


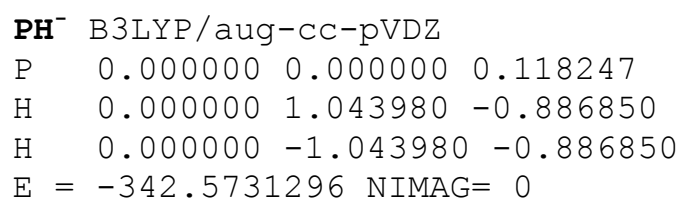

$\begin{array}{llll}\text { R-3 } & \text { CHSOPH } & \text { HF } / 6-31+G^{*} \\ \text { S } & 0.486173 & -0.239403 & -0.450406 \\ \mathrm{C} & 1.215776 & 1.280953 & 0.191445 \\ \mathrm{H} & 0.837838 & 2.136188 & -0.354608 \\ \mathrm{H} & 1.008518 & 1.374638 & 1.249893 \\ \mathrm{H} & 2.284243 & 1.194281 & 0.046635 \\ \mathrm{O} & 0.979592 & -1.339286 & 0.428706 \\ \mathrm{P} & -1.569110 & 0.217955 & 0.041438 \\ \mathrm{H} & -2.048921 & -1.038196 & -0.340317 \\ \mathrm{H} & -1.455173 & -0.077225 & 1.404995 \\ \mathrm{E}= & -853.801427400 & \mathrm{NIMAG}=0\end{array}$
R-3 $\mathrm{CH}_{3} \mathrm{SOPH} \quad \mathrm{MP}(\mathrm{FC}) / 6-31+\mathrm{G} *$
S $\quad 0.509545-0.231291-0.459485$
C $\quad 1.1099481 .3515850 .191966$
$\mathrm{H} \quad 0.647793 \quad 2.181429-0.349933$
$\mathrm{H} \quad 0.897358 \quad 1.414278 \quad 1.262847$
$\mathrm{H} \quad 2.191069 \quad 1.3605690 .039310$
O $\quad 1.089299-1.311242 \quad 0.440564$
P $\quad-1.592348 \quad 0.157900 \quad 0.037613$
$\mathrm{H} \quad-1.980785-1.168041-0.262543$
$\mathrm{H} \quad-1.397003 \quad-0.075659 \quad 1.421580$
$\mathrm{E}=-854.3593831 \mathrm{NIMAG}=0$

IDC-3 $\mathrm{HF} / 6-31+\mathrm{G}$ *

$$
\begin{array}{lrll}
\mathrm{S} & -1.291946 & -0.394336 & -0.472749 \\
\mathrm{P} & 3.247323 & -0.106803 & -0.149527 \\
\mathrm{C} & -0.521594 & -1.473833 & 0.741158 \\
\mathrm{H} & 0.557207 & -1.379546 & 0.676126 \\
\mathrm{H} & -0.885377 & -1.218493 & 1.728543 \\
\mathrm{H} & -0.823126 & -2.483905 & 0.492766 \\
\mathrm{O} & -2.774809 & -0.428667 & -0.209478 \\
\mathrm{P} & -0.622682 & 1.500071 & 0.348831 \\
\mathrm{H} & 0.674764 & 1.444688 & -0.168752 \\
\mathrm{H} & -1.181144 & 2.244610 & -0.700272 \\
\mathrm{H} & 4.030491 & -0.846413 & -1.074348 \\
\mathrm{H} & 4.256742 & -0.078245 & 0.849222 \\
\mathrm{E} & -1195.6753342 & -11 M A G=0
\end{array}
$$

$$
\begin{array}{lrll}
\text { IDC-3 } & \mathrm{MP}(\mathrm{FC}) / 6-31+\mathrm{G}^{*} \\
\mathrm{~S} & -0.944970 & -0.355314 & -0.464633 \\
\mathrm{P} & 2.477135 & -0.430358 & -0.151807 \\
\mathrm{C} & -0.744111 & -1.349445 & 1.041309 \\
\mathrm{H} & 0.314478 & -1.411839 & 1.297748 \\
\mathrm{H} & -1.336647 & -0.888832 & 1.837794 \\
\mathrm{H} & -1.147880 & -2.339271 & 0.813385 \\
\mathrm{O} & -2.473442 & -0.192348 & -0.595836 \\
\mathrm{P} & -0.050031 & 1.476358 & 0.412612 \\
\mathrm{H} & 0.349776 & 2.066693 & -0.806153 \\
\mathrm{H} & -1.359008 & 2.073871 & 0.389718 \\
\mathrm{H} & 2.962096 & -0.675217 & -1.474398 \\
\mathrm{H} & 3.182356 & 0.805071 & -0.017209 \\
\mathrm{E} & = & -1196.34900314 & \mathrm{NIMAG}=0
\end{array}
$$

$$
\begin{aligned}
& \text { IDC-3 B3LYP/6-31+G* } \\
& \text { S } \quad-0.989899-0.340469-0.465975 \\
& 2.526730-0.496099-0.164923 \\
& -0.839619-1.3716721 .056597 \\
& 0.215655-1.4626441 .319598 \\
& -1.427143-0.9034841 .853093 \\
& \begin{array}{llll}
-1.266596 & -2.348587 & 0.810310
\end{array} \\
& -2.507569-0.126141-0.624180 \\
& 0.029051 \quad 1.490628 \quad 0.442883 \\
& 0.4638592 .050372-0.788020 \\
& \begin{array}{lll}
-1.266514 & 2.149795 & 0.349231
\end{array} \\
& 2.694135-0.488057-1.593953 \\
& 3.1865490 .771339-0.010219 \\
& E=-1198.4092787 \mathrm{NIMAG}=0
\end{aligned}
$$

\footnotetext{
R-3 $\mathrm{CH}_{3} \mathrm{SOPH} \quad \mathrm{B} 3 \mathrm{LYP} / \mathrm{aug}-\mathrm{CC}-\mathrm{pVDZ}$

$\begin{array}{lllll}\text { S } & 0.529918 & -0.236589 & -0.469877\end{array}$

C $\quad 1.165658 \quad 1.362152 \quad 0.203623$

H $\quad 0.728443 \quad 2.192956-0.364108$

H $\quad 0.924467 \quad 1.429988 \quad 1.270821$

$\mathrm{H} \quad 2.252244 \quad 1.329694 \quad 0.063872$

$\begin{array}{lllll}0 & 1.092207 & -1.332994 & 0.450491\end{array}$

$\mathrm{P} \quad-1.644781 \quad 0.175623 \quad 0.040325$

$\mathrm{H} \quad-2.004282-1.166147-0.305778$

$\mathrm{H} \quad-1.439440 \quad-0.144365 \quad 1.422679$

$\mathrm{E}=-855.8870935 \mathrm{NIMAG}=0$
} 
IDC-3 B3LYP/aug-cC-pVDZ

$\begin{array}{lrll}\mathrm{S} & -0.981735 & -0.347991 & -0.466530 \\ \mathrm{P} & 2.531801 & -0.507126 & -0.168129 \\ \mathrm{C} & -0.841823 & -1.371618 & 1.066393 \\ \mathrm{H} & 0.216897 & -1.451692 & 1.332569 \\ \mathrm{H} & -1.443199 & -0.891734 & 1.848771 \\ \mathrm{H} & -1.264712 & -2.351856 & 0.814302 \\ \mathrm{O} & -2.520358 & -0.137222 & -0.623647 \\ \mathrm{P} & 0.027055 & 1.509252 & 0.441080 \\ \mathrm{H} & 0.465966 & 2.046450 & -0.808976 \\ \mathrm{H} & -1.284872 & 2.151474 & 0.322584 \\ \mathrm{H} & 2.672155 & -0.404437 & -1.602096 \\ \mathrm{H} & 3.176486 & 0.765232 & 0.053871 \\ \mathrm{E} & = & -1198.48502161 & \mathrm{NIMAG}=0\end{array}$

$\begin{array}{lrrr}\text { TS-3 HF/6-31+G* } \\ \text { S } 0.000000 & 0.153812 & 0.000000 \\ \mathrm{P} & -1.375739 & 0.722347 & 0.000000 \\ \mathrm{C} & -0.215300 & -1.645221 & 0.000000 \\ \mathrm{H} & -0.765446 & -1.924938 & 0.885162 \\ \mathrm{H} & -0.765446 & -1.924938 & -0.885162 \\ \mathrm{H} & 0.763362 & -2.114468 & 0.000000 \\ \mathrm{O} & 0.299003 & 0.148758 & -2.592939 \\ \mathrm{P} & 0.299003 & 0.148758 & 2.592939 \\ \mathrm{H} & 1.709912 & 0.008831 & 2.587352 \\ \mathrm{H} & 0.337658 & 1.557756 & 2.588779 \\ \mathrm{H} & 1.709912 & 0.008831 & -2.587352 \\ \mathrm{H} & 0.337658 & 1.557756 & -2.588779 \\ \mathrm{E}= & -1195.6433534 & \mathrm{NIMAG}= & 1\end{array}$

TS-3 MP (FC) / 6-31+G*

$\begin{array}{rrrr}\mathrm{S} & -0.362802 & 0.335908 & -0.191070 \\ \mathrm{O} & -0.632410 & 1.823854 & 0.006242 \\ \mathrm{P} & -2.448160 & -0.637037 & -0.257228 \\ \mathrm{P} & 3.046177 & -0.298218 & -0.182593 \\ \mathrm{C} & -0.035648 & -0.309002 & 1.467736 \\ \mathrm{H} & 0.930588 & 0.095623 & 1.768461 \\ \mathrm{H} & -0.845191 & 0.011242 & 2.129825 \\ \mathrm{H} & 0.046948 & -1.399315 & 1.423956 \\ \mathrm{H} & 3.429731 & -1.410724 & -0.996260 \\ \mathrm{H} & 3.211524 & 0.644917 & -1.242144 \\ \mathrm{H} & -1.979251 & -1.895694 & -0.726342 \\ \mathrm{H} & -2.686608 & -0.128581 & -1.559405 \\ \mathrm{E} & = & -1196.3353085 \mathrm{NIMAG}= & 1\end{array}$

TS-3 B3LYP/ 6-31+G*

$\begin{array}{rrrr}\mathrm{S} & -0.478974 & 0.542201 & -0.335035 \\ \mathrm{O} & -1.334014 & 1.770452 & -0.661671 \\ \mathrm{P} & -1.955870 & -1.295894 & 0.079445 \\ \mathrm{P} & 2.999811 & -0.452399 & -0.084931 \\ \mathrm{C} & -0.077371 & 0.741531 & 1.448698 \\ \mathrm{H} & 0.588234 & 1.605098 & 1.517413 \\ \mathrm{H} & -1.012320 & 0.918857 & 1.991337 \\ \mathrm{H} & 0.456905 & -0.142773 & 1.799815 \\ \mathrm{H} & 2.946032 & -0.796748 & -1.479887 \\ \mathrm{H} & 3.392303 & 0.902520 & -0.372272 \\ \mathrm{H} & -0.907845 & -2.246343 & -0.113578 \\ \mathrm{H} & -2.322499 & -1.304236 & -1.298791 \\ \mathrm{E} & =-1198.40165188 \mathrm{NIMAG}=1\end{array}$

TS-3 B3LYP/aug-CC-pVDZ
$\begin{array}{lrrr} \\ \mathrm{S} & -0.495535 & 0.542177 & -0.344163 \\ \mathrm{O} & -1.397540 & 1.773018 & -0.625790 \\ \mathrm{P} & -1.934547 & -1.310621 & 0.087915 \\ \mathrm{P} & 2.991761 & -0.463826 & -0.118441 \\ \mathrm{C} & -0.042792 & 0.733314 & 1.432521 \\ \mathrm{H} & 0.624083 & 1.600760 & 1.477821 \\ \mathrm{H} & -0.969307 & 0.902251 & 1.996632 \\ \mathrm{H} & 0.505570 & -0.158427 & 1.751887 \\ \mathrm{H} & 3.108428 & -0.323749 & -1.550611 \\ \mathrm{H} & 3.398170 & 0.905343 & 0.108863 \\ \mathrm{H} & -0.855639 & -2.239053 & -0.110233 \\ \mathrm{H} & -2.303888 & -1.329281 & -1.298667 \\ \mathrm{E} & = & -1198.47788240 \mathrm{NIMAG}=1\end{array}$

INT-3 MP (FC) / 6-31+G*

$\begin{array}{lrrr}\mathrm{S} & 0.000000 & 0.192737 & 0.000000 \\ \mathrm{O} & -1.351447 & 0.912544 & 0.000000 \\ \mathrm{C} & -0.412125 & -1.579719 & 0.000000 \\ \mathrm{H} & -0.987762 & -1.795597 & 0.901261 \\ \mathrm{H} & -0.987762 & -1.795597 & -0.901261 \\ \mathrm{H} & 0.520989 & -2.157113 & 0.000000 \\ \mathrm{P} & 0.347851 & 0.066225 & -2.645385 \\ \mathrm{P} & 0.347851 & 0.066225 & 2.645385 \\ \mathrm{H} & 1.770814 & -0.058154 & 2.587815 \\ \mathrm{H} & 0.380845 & 1.486008 & 2.578614 \\ \mathrm{H} & 1.770814 & -0.058154 & -2.587815 \\ \mathrm{H} & 0.380845 & 1.486008 & -2.578614 \\ \mathrm{E} & = & -1196.3428713 \mathrm{NIMAG}= & 0\end{array}$


INT-3 B $3 L Y P / 6-31+G$ *

$\begin{array}{lrrr}\mathrm{S} & -0.178163 & -0.114265 & 0.000000 \\ \mathrm{O} & -0.493227 & -1.607905 & 0.000000 \\ \mathrm{C} & 1.669162 & 0.000394 & 0.000000 \\ \mathrm{H} & 2.037407 & -0.492610 & 0.901571 \\ \mathrm{H} & 2.037407 & -0.492610 & -0.901571 \\ \mathrm{H} & 1.952272 & 1.059570 & 0.000000 \\ \mathrm{P} & -0.178163 & 0.361328 & -2.688838 \\ \mathrm{P} & -0.178163 & 0.361328 & 2.688838 \\ \mathrm{H} & -0.364952 & 1.777458 & 2.527899 \\ \mathrm{H} & -1.585426 & 0.110016 & 2.650209 \\ \mathrm{H} & -0.364952 & 1.777458 & -2.527899 \\ \mathrm{H} & -1.585426 & 0.110016 & -2.650209 \\ \mathrm{E} & =-1198.40832403 & \text { NIMAG } & 0\end{array}$

INT-3 B3LYP/aug-cC-pVDZ

S $0.000000 \quad 0.2174350 .000000$

O $-1.156452 \quad 1.243493 \quad 0.000000$

C $\quad-0.831051-1.4413390 .000000$

$\mathrm{H} \quad-1.440194-1.508396 \quad 0.906412$

$\mathrm{H} \quad-1.440194-1.508396-0.906412$

$\mathrm{H} \quad-0.046978-2.211470 \quad 0.000000$

P $\quad 0.395226-0.026579-2.678453$

P $\quad 0.395226-0.0265792 .678453$

$\mathrm{H} \quad 1.678210 \quad-0.664077 \quad 2.499298$

$\mathrm{H} \quad 0.976048 \quad 1.287453 \quad 2.628215$

$\mathrm{H} \quad 1.678210 \quad-0.664077 \quad-2.499298$

$\mathrm{H} \quad 0.976048 \quad 1.287453-2.628215$

$\mathrm{E}=-1198.4847542 \mathrm{NIMAG}=0$

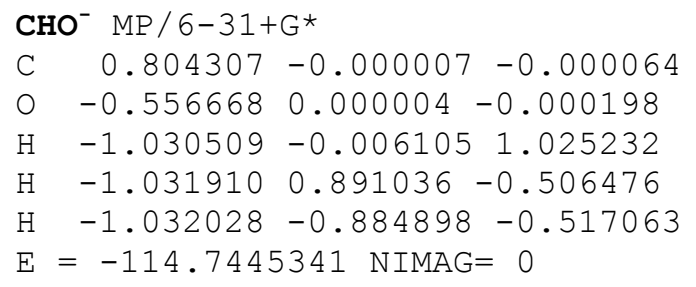

$\mathrm{CH}_{3} \mathrm{O}^{-}$B3LYP/6-31+G*

C $\quad-0.796574 \quad 0.000006-0.000085$

O $0.5435210 .000006-0.000207$

$\mathrm{H} \quad 1.035954 \quad-0.012979 \quad 1.030398$

$\mathrm{H} \quad 1.037706-0.885692-0.525452$

$\mathrm{H} \quad 1.0378020 .898585-0.503030$

$\mathrm{E}=-115.1115304 \mathrm{NIMAG}=0$

$\mathrm{CH}_{3} \mathrm{O}^{-}$B3LYP/aug-cC-pVDZ

$\mathrm{C} \quad-0.797098 \quad 0.000006-0.000111$

$0 \quad 0.5438240 .000013-0.000140$

$\mathrm{H} \quad 1.036626 \quad-0.070217 \quad 1.029859$

$\mathrm{H} \quad 1.038617-0.856482-0.574831$

$\mathrm{H} \quad 1.038598 \quad 0.926574 \quad-0.453303$

$\mathrm{E}=-115.1265593 \mathrm{NIMAG}=0$
R-4 ( $\mathrm{CHSOCH}) \mathrm{MP} / 6-31+\mathrm{G}^{\star}$

$\begin{array}{lllll}\mathrm{S} & -0.416581 & 0.226457 & -0.429662\end{array}$

$\begin{array}{lllll}0 & -0.584117 & 1.477766 & 0.375582\end{array}$

C $\quad-1.640619-0.937900 \quad 0.178422$

$\mathrm{H} \quad-1.471411-1.917794-0.272432$

$\mathrm{H} \quad-1.541780 \quad-0.981712 \quad 1.265524$

$\mathrm{H} \quad-2.623983-0.550184-0.098367$

$\begin{array}{lllll}0 & 0.834067 & -0.723021 & 0.217525\end{array}$

$\begin{array}{llll}\text { C } \quad 2.130284 & -0.108732 & 0.030328\end{array}$

$\mathrm{H} \quad 2.844832-0.825828 \quad 0.432360$

$\mathrm{H} \quad 2.329702 \quad 0.061894 \quad-1.033237$

$\mathrm{H} \quad 2.190347 \quad 0.832138 \quad 0.583385$

$\mathrm{E}=-627.1670142 \mathrm{NIMAG}=0$

R-4 ( $\mathrm{CHSOCH)} \mathrm{B3LYP/6-31+G*}$

S $\quad-0.416411 \quad 0.234296-0.426858$

O $\quad-0.611741 \quad 1.480337 \quad 0.377786$

C $\quad-1.653491-0.965807 \quad 0.167795$

$\mathrm{H} \quad-1.482519-1.932864-0.311704$

$\mathrm{H} \quad-1.552470-1.040238 \quad 1.253657$

$\mathrm{H} \quad-2.636194-0.568749-0.100006$

$\begin{array}{lllll}0 & 0.848833 & -0.692073 & 0.237481\end{array}$

C $\quad 2.150351-0.121072 \quad 0.021054$

$\mathrm{H} \quad 2.857188-0.835616 \quad 0.447660$

$\mathrm{H} \quad 2.355382 \quad 0.002603-1.050226$

$\mathrm{H} \quad 2.243305 \quad 0.841296 \quad 0.535118$

$\mathrm{E}=-628.4152449 \mathrm{NIMAG}=0$

R-4 (CHSOCH) B3LYP/aug-cC-pVDZ

S $\quad-0.419352 \quad 0.228451-0.435158$

O $\quad-0.6176161 .487255 \quad 0.383096$

C $\quad-1.660260-0.967785 \quad 0.171994$

$\mathrm{H} \quad-1.487659-1.936113-0.311227$

$\mathrm{H} \quad-1.547519-1.030314 \quad 1.259958$

$\mathrm{H} \quad-2.639850-0.558996-0.100571$

$\begin{array}{lllll}0 & 0.861121 & -0.696720 & 0.251096\end{array}$

C $\quad 2.154472-0.1146690 .016861$

$\mathrm{H} \quad 2.875198-0.803440 \quad 0.471393$

$\mathrm{H} \quad 2.362225-0.026264-1.060757$

$\mathrm{H} \quad 2.233927 \quad 0.870353 \quad 0.497058$

$\mathrm{E}=-628.4552453 \mathrm{NIMAG}=0$ 
IDC-4 MP / 6-31+G*

$\begin{array}{lrll}\mathrm{S} & -0.639462 & 0.167175 & -0.259028 \\ \mathrm{O} & -0.728263 & 1.515964 & 0.407506 \\ \mathrm{C} & 0.421553 & -0.861389 & 0.713655 \\ \mathrm{H} & 0.121739 & -1.901566 & 0.557105 \\ \mathrm{H} & 1.480768 & -0.720266 & 0.313218 \\ \mathrm{H} & 0.291887 & -0.564786 & 1.758851 \\ \mathrm{O} & -2.081108 & -0.715942 & 0.169644 \\ \mathrm{O} & 2.961181 & -0.617308 & -0.530910 \\ \mathrm{C} & 3.771482 & 0.370164 & -0.019785 \\ \mathrm{H} & 4.743335 & -0.006996 & 0.388214 \\ \mathrm{H} & 4.057384 & 1.153190 & -0.765090 \\ \mathrm{H} & 3.305944 & 0.939115 & 0.828835 \\ \mathrm{C} & -3.247029 & -0.060144 & -0.344256 \\ \mathrm{H} & -3.202186 & 0.030989 & -1.438189 \\ \mathrm{H} & -4.092608 & -0.692321 & -0.065466 \\ \mathrm{H} & -3.365392 & 0.934345 & 0.099369 \\ \mathrm{E} & = & -741.9417867 & \mathrm{NIMAG}=0\end{array}$

IDC-4 B3LYP/6-31+G*

\begin{tabular}{|c|c|c|}
\hline$S$ & -0.701464 & $0.198263-0.302288$ \\
\hline O & -0.810722 & $1.567790 \quad 0.318790$ \\
\hline $\mathrm{C}$ & 0.484605 & $-0.756464 \quad 0.631264$ \\
\hline $\mathrm{H}$ & 0.236926 & $\begin{array}{lll}-1.814952 & 0.498897\end{array}$ \\
\hline $\mathrm{H}$ & 1.543286 & -0.5803130 .187169 \\
\hline & 0.399514 & $-0.459112 \quad 1.681814$ \\
\hline O & -2.099342 & -0.7214320 .241393 \\
\hline & 3.018237 & $-0.574482-0.522729$ \\
\hline $\mathrm{C}$ & 3.915522 & 0.2759650 .048043 \\
\hline & 4.830691 & -0.2295770 .467813 \\
\hline & 4.324005 & $1.055218-0.653433$ \\
\hline 7 & 3.503348 & 0.8703790 .913742 \\
\hline & -3.320918 & $-0.186621-0.252403$ \\
\hline $\mathrm{H}$ & -3.347218 & -0.181122 \\
\hline & -4.118785 & $-0.839354 \quad 0.118234$ \\
\hline & -3.48 & 0.8343360 .115290 \\
\hline & 7 & 384 NIMAG $=0$ \\
\hline
\end{tabular}

TS-4 MP / 6-31+G*

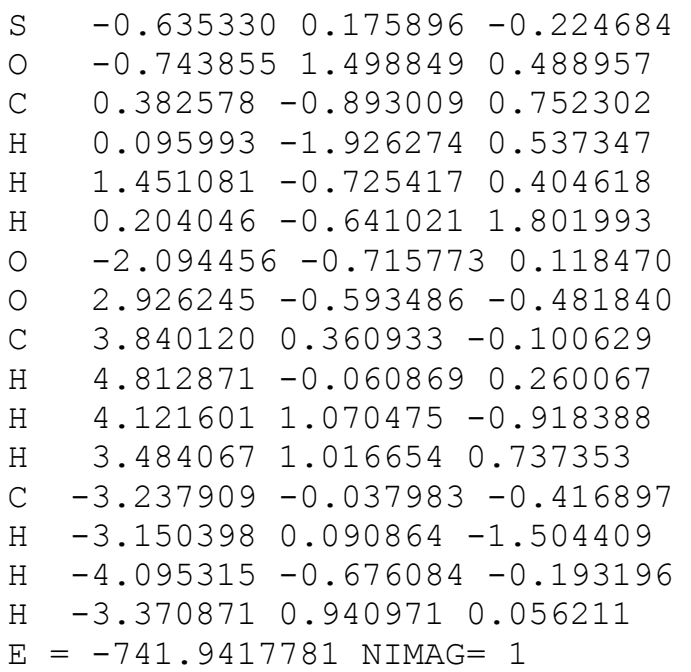

TS-4 B3LYP/ 6-31+G*

$\begin{array}{lrll}\mathrm{S} & -0.582361 & 0.207339 & -0.106065 \\ \mathrm{O} & -0.776328 & 1.443258 & 0.732832 \\ \mathrm{C} & 0.310753 & -0.995973 & 0.891294 \\ \mathrm{H} & 0.039940 & -1.996901 & 0.542807 \\ \mathrm{H} & 1.393294 & -0.785599 & 0.667776 \\ \mathrm{H} & 0.016678 & -0.839446 & 1.934000 \\ \mathrm{O} & -2.093822 & -0.697187 & -0.026152 \\ \mathrm{O} & 2.722814 & -0.376043 & -0.416005 \\ \mathrm{C} & 3.921797 & 0.251520 & -0.310148 \\ \mathrm{H} & 4.808261 & -0.443834 & -0.229615 \\ \mathrm{H} & 4.174129 & 0.915392 & -1.185584 \\ \mathrm{H} & 4.020167 & 0.928650 & 0.587456 \\ \mathrm{C} & -3.163308 & -0.009321 & -0.660140 \\ \mathrm{H} & -2.950204 & 0.184265 & -1.723761 \\ \mathrm{H} & -4.040622 & -0.661553 & -0.587732 \\ \mathrm{H} & -3.380622 & 0.944024 & -0.159742 \\ \mathrm{E} & = & -743.5550665 & \text { NIMAG }=1\end{array}$

TS-4 B3LYP/aug-cC-pVDZ

$\mathrm{S} \quad-0.545298 \quad 0.191959-0.085627$

O $\quad-0.731299 \quad 1.392900 \quad 0.834501$

C $\quad 0.313845-1.080200 \quad 0.861950$

H $\quad 0.020016-2.053543 \quad 0.450565$

$\mathrm{H} \quad 1.401014-0.853886 \quad 0.640707$

$\mathrm{H} \quad 0.003345-0.963758 \quad 1.907312$

o $\quad-2.099837-0.685694-0.064929$

o $\quad 2.634951-0.313884-0.426332$

C $\quad 3.862477 \quad 0.264306-0.329935$

$\mathrm{H} \quad 4.725864-0.463887-0.373244$

$\mathrm{H} \quad 4.088340 \quad 1.006359-1.149466$

$\mathrm{H} \quad 4.036326 \quad 0.844265 \quad 0.623819$

C $\quad-3.130765 \quad 0.067307-0.686683$

$\mathrm{H} \quad-2.899518 \quad 0.284285-1.745519$

$\mathrm{H} \quad-4.043313-0.544444-0.642909$

$\mathrm{H} \quad-3.311178 \quad 1.018214-0.159149$

$\mathrm{E}=-743.6101404 \mathrm{NIMAG}=1$

\begin{tabular}{|c|c|c|}
\hline \multicolumn{3}{|c|}{ INT-4 MP/6-31+G* } \\
\hline S & 0.000000 & $0.081940 \quad 0.000000$ \\
\hline O & 0.926092 & $-1.121470 \quad 0.000000$ \\
\hline C & 1.046225 & 1.5563610 .000000 \\
\hline $\mathrm{H}$ & 0.407393 & 2.4436030 .000000 \\
\hline $\mathrm{H}$ & 1.649759 & 1.520934 \\
\hline $\mathrm{H}$ & 1.649759 & -0.907449 \\
\hline O & -0.091183 & $0.400944-1.936889$ \\
\hline O & -0.091183 & $0.400944 \quad 1.936889$ \\
\hline $\mathrm{C}$ & -0.814881 & -0.6568722 .513218 \\
\hline $\mathrm{H}$ & -1.176177 & -0.3515473 .507091 \\
\hline $\mathrm{H}$ & -1.699538 & -0.9299721 .903470 \\
\hline $\mathrm{H}$ & -0.20 & -1.5662572 .628899 \\
\hline $\mathrm{C}$ & -0.814881 & $-0.656872-2.513218$ \\
\hline $\mathrm{H}$ & -1.69 & $-0.929972-1.903470$ \\
\hline $\mathrm{H}$ & -1.176177 & $-0.351547-3.507091$ \\
\hline $\mathrm{H}$ & -0.20 & $-1.566257-2.628899$ \\
\hline & $-7<$ & 5096 NIMAG $=0$ \\
\hline
\end{tabular}


INT-4 B3LYP/ 6-31+G*

S $\quad 0.000000 \quad 0.0533390 .000000$

o $\quad 1.131216-0.9535190 .000000$

C $\quad 0.7691201 .7202260 .000000$

$\mathrm{H} \quad-0.027394 \quad 2.470274 \quad 0.000000$

$\mathrm{H} \quad 1.366414 \quad 1.795653 \quad 0.909032$

$\mathrm{H} \quad 1.366414 \quad 1.795653-0.909032$

$\begin{array}{lllll}0 & -0.158587 & 0.364869 & -1.969860\end{array}$

$\begin{array}{lllll}0 & -0.158587 & 0.364869 & 1.969860\end{array}$

C $\quad-0.711466-0.7502952 .588002$

$\mathrm{H} \quad-1.089734 \quad-0.477089 \quad 3.591221$

$\mathrm{H} \quad-1.574127-1.168764 \quad 2.022254$

$\mathrm{H} \quad 0.016408 \quad-1.575436 \quad 2.718432$

C $\quad-0.711466-0.750295-2.588002$

$\mathrm{H} \quad-1.574127-1.168764 \quad-2.022254$

$\mathrm{H} \quad-1.089734-0.477089-3.591221$

H $\quad 0.016408-1.575436-2.718432$

$\mathrm{E}=-743.5729125 \mathrm{NIMAG}=0$

INT-4 B3LYP/aug-cC-pVDZ

S $\quad 0.000000 \quad 0.0555610 .000000$

o $\quad 1.246305-0.833950 \quad 0.000000$

C $\quad 0.6080311 .7909710 .000000$

$\mathrm{H} \quad-0.261434 \quad 2.459350 \quad 0.000000$

$\mathrm{H} \quad 1.195871 \quad 1.912203 \quad 0.913188$

$\mathrm{H} \quad 1.195871 \quad 1.912203-0.913188$

$\begin{array}{lllll}0 & -0.184553 & 0.343914 & -1.973524\end{array}$

$\begin{array}{lllll}0 & -0.184553 & 0.343914 & 1.973524\end{array}$

C $\quad-0.660841-0.816612 \quad 2.572612$

$\mathrm{H} \quad-1.145134 \quad-0.579288 \quad 3.542231$

$\mathrm{H} \quad-1.427462-1.334455 \quad 1.949170$

H $\quad 0.139606 \quad-1.561364 \quad 2.773110$

C $\quad-0.660841-0.816612-2.572612$

$\mathrm{H} \quad-1.427462-1.334455-1.949170$

$\mathrm{H} \quad-1.145134-0.579288-3.542231$

$\mathrm{H} \quad 0.139606-1.561364-2.773110$

$\mathrm{E}=-743.6282699 \mathrm{NIMAG}=0$

HO- $^{-} \mathrm{MP} / 6-31+\mathrm{G}$ *

$0 \quad 0.000000 \quad 0.000000 \quad 0.108615$

$\mathrm{H} \quad 0.000000 \quad 0.000000-0.868919$

$\mathrm{E}=-75.5883641 \mathrm{NIMAG}=0$

HO- B3LYP/6-31+G*

O $0.000000 \quad 0.000000 \quad 0.108339$

$\mathrm{H} \quad 0.000000 \quad 0.000000-0.866716$

$\mathrm{E}=-75.7966809 \mathrm{NIMAG}=0$

HO- B3LYP/aug-cC-pVDZ

$0 \quad 0.000000 \quad 0.000000 \quad 0.107757$

$\mathrm{H} \quad 0.000000 \quad 0.000000-0.862058$

$\mathrm{E}=-75.8169343 \mathrm{NIMAG}=0$
R-5 ( $\left.\mathrm{CH}_{3} \mathrm{SOH}\right) \quad \mathrm{MP} / 6-31+\mathrm{G}^{*}$

$\mathrm{S} \quad-0.170460-0.151845-0.423180$

$0 \quad-0.815175-1.234947 \quad 0.379266$

$\begin{array}{llll}0 & -0.506102 & 1.375636 & 0.259880\end{array}$

C $\quad 1.526464-0.040731 \quad 0.149595$

$\mathrm{H} \quad 1.499356 \quad 0.102755 \quad 1.232495$

H $\quad 2.014146-0.986641-0.095838$

$\mathrm{H} \quad 2.028929 \quad 0.793057 \quad-0.344384$

$\mathrm{H} \quad-1.403637 \quad 1.639219-0.032125$

$\mathrm{E}=-588.0105764 \mathrm{NIMAG}=0$

R-5 ( $\left.\mathrm{CH}_{3} \mathrm{SOH}\right)$ B3LYP/6-31+G*

$\mathrm{S} \quad-0.165713-0.160998-0.425160$

$\begin{array}{lllll}0 & -0.766458 & -1.263861 & 0.382651\end{array}$

$\begin{array}{lllll}0 & -0.581708 & 1.350887 & 0.256446\end{array}$

C $\quad 1.552804 \quad 0.027887 \quad 0.152108$

H $\quad 1.525669 \quad 0.185264 \quad 1.233706$

$\mathrm{H} \quad 2.073379-0.902791-0.087445$

$\mathrm{H} \quad 2.017377 \quad 0.875374-0.358023$

$\mathrm{H} \quad-1.4965091 .554592-0.011097$

$\mathrm{E}=-589.1087967 \mathrm{NIMAG}=0$

R-5 ( $\left.\mathrm{CH}_{3} \mathrm{SOH}\right) \quad \mathrm{B} 3 \mathrm{LYP} / \mathrm{aug}-\mathrm{CC}-\mathrm{pVDZ}$

S $-0.150605-0.168641-0.434406$

$0 \quad-0.684897-1.319055 \quad 0.388104$

$\begin{array}{lllll}0 & -0.677851 & 1.320951 & 0.264935\end{array}$

C $\quad 1.553177 \quad 0.1220890 .158776$

$\mathrm{H} \quad 1.498576 \quad 0.256763 \quad 1.244904$

$\mathrm{H} \quad 2.127047-0.774977-0.099514$

$\mathrm{H} \quad 1.9579561 .009648-0.340604$

$\mathrm{H} \quad-1.5909721 .459112-0.031259$

$\mathrm{E}=-589.1535392 \mathrm{NIMAG}=0$

IDC-5 MP / 6-31+G*

S $\quad-0.579244 \quad 0.193598-0.377805$

$\begin{array}{lllll}0 & -0.906739 & 1.442815 & 0.400693\end{array}$

C $\quad 0.541101-0.737676 \quad 0.634446$

$\mathrm{H} \quad 1.589992-0.339903 \quad 0.438882$

H $\quad 0.227236-0.614344 \quad 1.678013$

$\mathrm{H} \quad 0.502085-1.784292 \quad 0.320211$

o $\quad-1.953549-0.845541-0.259776$

$\begin{array}{lllll}0 & 3.129997 & -0.065071 & -0.229848\end{array}$

$\mathrm{H} \quad-2.160140 \quad-0.912620 \quad 0.698726$

$\mathrm{H} \quad 3.704444 \quad 0.722023-0.186182$

$E=-663.634215 \mathrm{NIMAG}=0$ 
TS-5 MP / 6-31+G*

$\begin{array}{lrll}\mathrm{S} & -0.510077 & 0.198315 & -0.356419 \\ \mathrm{O} & -0.853815 & 1.434883 & 0.435000 \\ \mathrm{O} & -1.929854 & -0.790280 & -0.361029 \\ \mathrm{O} & 2.997156 & -0.049954 & -0.276414 \\ \mathrm{H} & -2.229030 & -0.833194 & 0.574070 \\ \mathrm{H} & 3.653376 & 0.671589 & -0.265516 \\ \mathrm{C} & 0.490623 & -0.802870 & 0.718587 \\ \mathrm{H} & 1.545728 & -0.424704 & 0.587103 \\ \mathrm{H} & 0.102446 & -0.686677 & 1.737475 \\ \mathrm{H} & 0.437085 & -1.840031 & 0.377598 \\ \mathrm{E}= & -663.6341708 & \mathrm{NIMAG}= & 1\end{array}$

TS-5 B3LYP/6-31+G*

S $\quad-0.442771 \quad 0.207960-0.308336$

$\begin{array}{lllll}0 & -0.760976 & 1.444011 & 0.491985\end{array}$

C $\quad 0.468866-0.916416 \quad 0.755470$

$\mathrm{H} \quad 1.544314 \quad-0.583147 \quad 0.604974$

$\mathrm{H} \quad 0.077344 \quad-0.800862 \quad 1.771648$

H $\quad 0.326503-1.934410 \quad 0.380195$

$0 \quad-1.910873-0.780058-0.289479$

o $\quad 2.849903-0.030245-0.368792$

$\mathrm{H} \quad-2.552801-0.291784-0.835080$

$\mathrm{H} \quad 3.451345 \quad 0.711676-0.190893$

$\mathrm{E}=-664.9402066 \mathrm{NIMAG}=1$

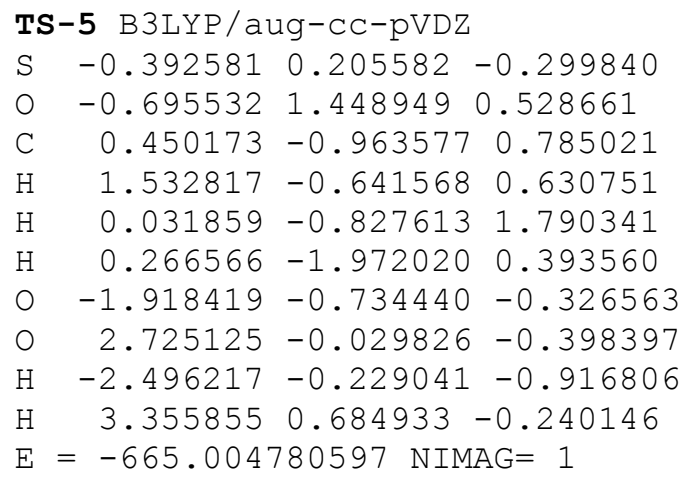

INT-5 MP/6-31+G*

S $\quad-0.008929-0.153122-0.341985$

$\begin{array}{lllll}0 & -0.021763 & -1.262016 & 0.723439\end{array}$

$0 \quad 1.928578-0.028886-0.337820$

$0 \quad-1.944186 \quad 0.027753-0.324488$

H $\quad 2.153669-0.828441 \quad 0.177911$

$\mathrm{H} \quad-2.196676-0.779221 \quad 0.166443$

C $\quad 0.0456401 .4783460 .460353$

$\mathrm{H} \quad-0.934642 \quad 1.9473890 .379603$

$\mathrm{H} \quad 0.306267 \quad 1.284009 \quad 1.505851$

$\mathrm{H} \quad 0.8393812 .061332-0.009209$

$E=-663.6536893 \mathrm{NIMAG}=0$

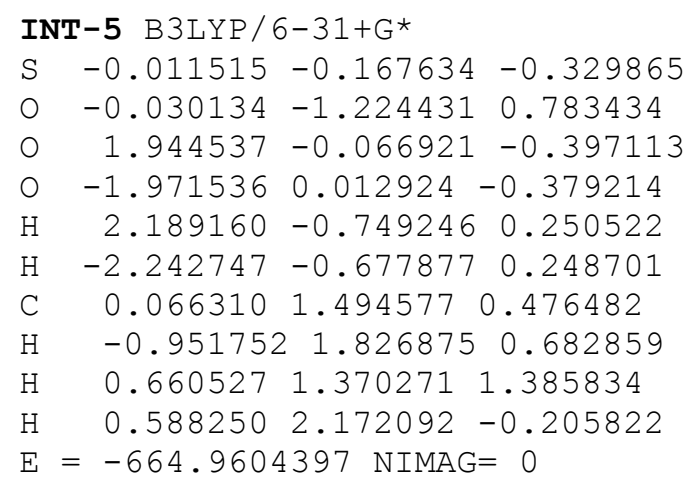

INT-5 B3LYP/aug-cC-pVDZ

$$
\begin{array}{lrll}
\mathrm{S} & 0.000000 & 0.368978 & 0.000000 \\
\mathrm{O} & -1.453261 & -0.173568 & 0.000000 \\
\mathrm{C} & 1.097347 & -1.122476 & 0.000000 \\
\mathrm{H} & 2.137095 & -0.767566 & 0.000000 \\
\mathrm{H} & 0.883859 & -1.678353 & 0.917511 \\
\mathrm{H} & 0.883859 & -1.678353 & -0.917511 \\
\mathrm{O} & 0.160389 & 0.389592 & -1.965512 \\
\mathrm{O} & 0.160389 & 0.389592 & 1.965512 \\
\mathrm{H} & -0.714519 & 0.055283 & -2.207793 \\
\mathrm{H} & -0.714519 & 0.055283 & 2.207793 \\
\mathrm{E} & =-665.0235667 & \mathrm{NIMAG}= & 0
\end{array}
$$

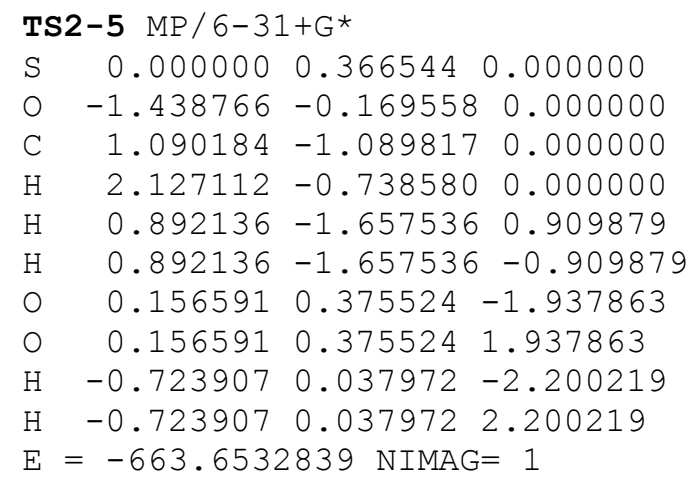

TS2-5 B3LYP/6-31+G*

$\begin{array}{lrll}\mathrm{S} & 0.000000 & 0.367217 & 0.000000 \\ \mathrm{O} & -1.445644 & -0.146287 & 0.000000 \\ \mathrm{C} & 1.088804 & -1.126599 & 0.000000 \\ \mathrm{H} & 2.129266 & -0.782348 & 0.000000 \\ \mathrm{H} & 0.883434 & -1.688968 & 0.912385 \\ \mathrm{H} & 0.883434 & -1.688968 & -0.912385 \\ \mathrm{O} & 0.161066 & 0.379880 & -1.957388 \\ \mathrm{O} & 0.161066 & 0.379880 & 1.957388 \\ \mathrm{H} & -0.720434 & 0.068314 & -2.225140 \\ \mathrm{H} & -0.720434 & 0.068314 & 2.225140 \\ \mathrm{E}= & -664.960364529 & \mathrm{NIMAG}=1\end{array}$




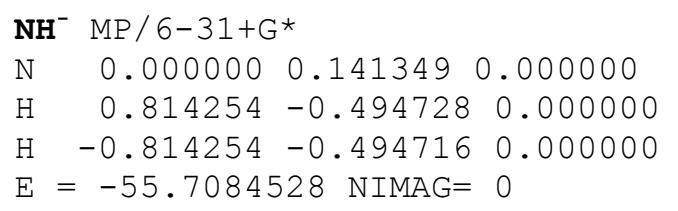
$\mathrm{NH}^{-}$B3LYP/ 6-31+G*
$\mathrm{N} \quad 0.000000 \quad 0.143216 \quad 0.000000$
$\mathrm{H} \quad 0.810207 \quad-0.501259 \quad 0.000000$
$\mathrm{H} \quad-0.810207-0.501253 \quad 0.000000$
$\mathrm{E}=-55.9006864 \mathrm{NIMAG}=0$
$\mathbf{N H}^{-}$B3LYP/aug-cC-pVDZ
$\mathrm{N} \quad 0.000000 \quad 0.144454 \quad 0.000000$
$\mathrm{H} \quad 0.804430 \quad-0.505586 \quad 0.000000$
$\mathrm{H} \quad-0.804430-0.505592 \quad 0.000000$
$\mathrm{E}=-55.9180538 \mathrm{NIMAG}=0$
R-6 ( $\left.\mathrm{CH}_{3} \mathrm{SONH}\right) \quad \mathrm{MP} / 6-31+\mathrm{G}$ *
$\mathrm{S} \quad-0.075322 \quad 0.204747 \quad-0.429491$
O $\quad-0.177272 \quad 1.473361 \quad 0.384111$
C $\quad 1.418912-0.6191520 .150156$
H $\quad 1.516624-1.591606-0.335367$
$\mathrm{H} \quad 2.262183 \quad 0.026697 \quad-0.102403$
$\mathrm{H} \quad 1.358690 \quad-0.724736 \quad 1.237640$
$\mathrm{N} \quad-1.118957-1.025119 \quad 0.155595$
$\mathrm{H} \quad-2.069285-0.844708-0.171688$
$\mathrm{H} \quad-1.125662-1.037742 \quad 1.180680$
$\mathrm{E}=-568.1627681 \mathrm{NIMAG}=0$

\section{R-6 ( $\left.\mathrm{CH}_{3} \mathrm{SONH}\right)$ B3LYP/6-31+G * \\ $\mathrm{S} \quad-0.084903 \quad 0.207207-0.430495$ \\ $\begin{array}{lllll}0 & -0.246138 & 1.464322 & 0.388703\end{array}$ \\ C $\quad 1.468477-0.565980 \quad 0.151315$ \\ $\mathrm{H} \quad 1.607606-1.526375-0.350149$ \\ $\mathrm{H} \quad 2.278102 \quad 0.124066-0.098413$ \\ $\mathrm{H} \quad 1.417350 \quad-0.691177 \quad 1.237874$ \\ $\mathrm{N} \quad-1.091070-1.076036 \quad 0.154084$ \\ $\mathrm{H} \quad-2.043950 \quad-0.921465-0.175148$ \\ $\mathrm{H} \quad-1.104922-1.0868051 .177648$ \\ $\mathrm{E}=-569.2404032 \mathrm{NIMAG}=0$}

\footnotetext{
R-6 ( $\left.\mathrm{CH}_{3} \mathrm{SONH}\right) \quad \mathrm{B} 3 \mathrm{LYP} / \mathrm{aug}-\mathrm{CC}-\mathrm{pVDZ}$

S $\quad-0.074621 \quad 0.201032-0.441515$

O $\quad-0.214775 \quad 1.476685 \quad 0.390404$

C $\quad 1.462528-0.596467 \quad 0.162354$

$\mathrm{H} \quad 1.581225-1.566734-0.332368$

$\mathrm{H} \quad 2.2822120 .083467-0.094286$

$\mathrm{H} \quad 1.388043 \quad-0.703952 \quad 1.251694$

$\mathrm{N} \quad-1.127878 \quad-1.057238 \quad 0.161720$

$\mathrm{H} \quad-2.069459-0.854395-0.174308$

$\mathrm{H} \quad-1.149902-1.008910 \quad 1.184127$

$\mathrm{E}=-569.280195 \mathrm{NIMAG}=0$
}

IDC- $6 \mathrm{MP} / 6-31+\mathrm{G}$ *

$$
\begin{aligned}
& \text { S } \quad-0.635685 \quad 0.194129-0.381983 \\
& \begin{array}{lllll}
0 & -0.962786 & 1.473433 & 0.375667
\end{array} \\
& \mathrm{~N} \quad-1.957899-0.929681-0.183491 \\
& \mathrm{~N} \quad 3.331130-0.011559-0.150266 \\
& \text { C } \quad 0.524813-0.726265 \quad 0.610401 \\
& \mathrm{H} \quad 1.569716-0.362475 \quad 0.392111 \\
& \mathrm{H} \quad 0.242762-0.575597 \quad 1.659442 \\
& \mathrm{H} \quad 0.459673-1.779749 \quad 0.324815 \\
& \mathrm{H} \quad 3.826629 \quad 0.886877-0.052881 \\
& \mathrm{H} \quad 3.957348-0.592675-0.727362 \\
& \mathrm{H} \quad-2.166617-1.007376 \quad 0.818191 \\
& \mathrm{H} \quad-2.777766-0.516271-0.634030 \\
& \mathrm{E}=-623.899982 \mathrm{NIMAG}=0
\end{aligned}
$$

$$
\begin{aligned}
& \text { IDC- } 6 \text { B3LYP/6-31+G* } \\
& \mathrm{S} \quad-0.633277 \quad 0.193325-0.380543 \\
& \text { O } \quad-0.943446 \quad 1.472282 \quad 0.386628 \\
& \text { C } \quad 0.575502-0.735359 \quad 0.581217 \\
& \mathrm{H} \quad 0.504466-1.787759 \quad 0.285772 \\
& \mathrm{H} \quad 1.646252-0.363418 \quad 0.333668 \\
& \mathrm{H} \quad 0.328164-0.599280 \quad 1.642148 \\
& \mathrm{~N} \quad-1.990873-0.928926-0.162407 \\
& \mathrm{~N} \quad 3.273289 \quad 0.018609-0.244270 \\
& \mathrm{H} \quad 4.014225-0.695068-0.300508 \\
& \mathrm{H} \quad 3.748240 \quad 0.846133 \quad 0.143720 \\
& \mathrm{H} \quad-2.808569-0.489686-0.592029 \\
& \mathrm{H} \quad-2.182690 \quad-0.998011 \quad 0.842324 \\
& E=-625.1692454 \mathrm{NIMAG}=0
\end{aligned}
$$

\footnotetext{
TS-6 MP / 6-31+G*

S $\quad-0.397916 \quad 0.201274-0.314209$

$\begin{array}{lllll}0 & -0.549738 & 1.410513 & 0.595897\end{array}$

$\mathrm{N} \quad-1.983840-0.560007-0.447822$

$\mathrm{N} \quad 2.832059-0.001274-0.273470$

C $\quad 0.293711-1.101208 \quad 0.696443$

$\mathrm{H} \quad 1.378638-0.863109 \quad 0.721161$

$\mathrm{H} \quad-0.203950-1.063689 \quad 1.673020$

$\mathrm{H} \quad 0.129248 \quad-2.058572 \quad 0.194908$

$\mathrm{H} \quad 3.198235 \quad 0.932480-0.033288$

$\mathrm{H} \quad 3.522099-0.378637-0.941351$

$\mathrm{H} \quad-2.385243-0.621596 \quad 0.495034$

$\mathrm{H} \quad-2.574274 \quad 0.084854 \quad-0.978928$

$\mathrm{E}=-623.8991676 \mathrm{NIMAG}=1$
} 
TS-6 B $3 \mathrm{LYP} / 6-31+\mathrm{G}$ *

S $\quad-0.399467 \quad 0.210246-0.312524$

O $\quad-0.593616 \quad 1.416436 \quad 0.593913$

C $\quad 0.333101-1.097519 \quad 0.711425$

$\mathrm{H} \quad 0.138037-2.062525 \quad 0.233165$

H $\quad 1.425195 \quad-0.858114 \quad 0.679508$

$\mathrm{H} \quad-0.123734-1.039833 \quad 1.707718$

$\mathrm{N} \quad-2.000457-0.593262-0.454608$

N $2.8532330 .001153-0.277843$

$\mathrm{H} \quad 3.469197-0.303716-1.046072$

$\mathrm{H} \quad 3.259290 \quad 0.899919 \quad 0.020365$

$\mathrm{H} \quad-2.594494 \quad 0.056622 \quad-0.974276$

$\mathrm{H} \quad-2.401140 \quad-0.657904 \quad 0.487279$

$\mathrm{E}=-625.1676375 \mathrm{NIMAG}=1$
INT-6 MP / 6-31+G*

S $\quad 0.000000 \quad 0.343487 \quad 0.000000$

$0 \quad 1.468624-0.143403 \quad 0.000000$

$\mathrm{N} \quad-0.217144 \quad 0.339827 \quad 1.992220$

$\mathrm{N} \quad-0.217144 \quad 0.339827-1.992220$

C $\quad-1.035632-1.143484 \quad 0.000000$

$\mathrm{H} \quad-0.812636-1.710631-0.905299$

$\mathrm{H} \quad-0.812636-1.7106310 .905299$

$\mathrm{H} \quad-2.085471-0.839615 \quad 0.000000$

$\mathrm{H} \quad 0.523133-0.279328-2.350461$

$\mathrm{H} \quad 0.084649 \quad 1.287147 \quad-2.251477$

H $\quad 0.523133 \quad-0.279328 \quad 2.350461$

$\mathrm{H} \quad 0.084649 \quad 1.287147 \quad 2.251477$

$\mathrm{E}=-623.9123953 \mathrm{NIMAG}=0$

\footnotetext{
INT-6

B3LYP/aug-cC-pVDZ

S $\quad 0.000000 \quad 0.3462850 .000000$

$\begin{array}{llll}0 & 1.483982 & -0.134271 & 0.000000\end{array}$

$\mathrm{N} \quad-0.233302 \quad 0.347255 \quad 2.062211$

$\mathrm{N} \quad-0.233302 \quad 0.347255-2.062211$

C $-1.031705-1.181596 \quad 0.000000$

$\mathrm{H} \quad-0.797963-1.739025-0.911839$

$\mathrm{H} \quad-0.797963-1.739025 \quad 0.911839$

$\mathrm{H} \quad-2.084909-0.874615 \quad 0.000000$

$\mathrm{H} \quad 0.548694 \quad-0.239546-2.376825$

$\mathrm{H} \quad 0.084023 \quad 1.296683-2.286819$

$\mathrm{H} \quad 0.548694 \quad-0.239546 \quad 2.376825$

$\mathrm{H} \quad 0.084023 \quad 1.296683 \quad 2.286819$

$E=-625.2360053 \mathrm{NIMAG}=0$
}

\begin{tabular}{|c|c|c|c|}
\hline \multicolumn{4}{|c|}{ INT-6 B3LYP/6-31+G* } \\
\hline $\mathrm{S}$ & 0.000000 & 0.343987 & 0.000000 \\
\hline 0 & 1.480751 & -0.089676 & 60.000000 \\
\hline $\mathrm{N}$ & -0.238639 & 0.3415822 & 2.037131 \\
\hline $\mathrm{N}$ & -0.238639 & 0.341582 & -2.037131 \\
\hline C & -1.010685 & -1.194493 & 30.000000 \\
\hline $\mathrm{H}$ & -0.777199 & -1.756853 & $3-0.90640^{\circ}$ \\
\hline $\mathrm{H}$ & -0.777199 & -1.756853 & 30.906407 \\
\hline $\mathrm{H}$ & -2.066333 & -0.908356 & 60.000000 \\
\hline $\mathrm{H}$ & 0.504817 & -0.273779 & $9-2.39128$ \\
\hline $\mathrm{H}$ & 0.085071 & 1.284022 & -2.283975 \\
\hline $\mathrm{H}$ & 0.504817 & -0.273779 & 92.391287 \\
\hline $\mathrm{H}$ & 0.085071 & 1.284022 & 2.283975 \\
\hline & $=-625.181$ & 4787 NIMAG & $G=0$ \\
\hline
\end{tabular}

